Rev. Biol. Trop., 47(4): 887-911, 1999

www.ucr.ac.cr www.ots.ac.cr www.ots.duke.edu

\title{
Biodiversidad marina de Costa Rica: Crustacea: Decapoda (Penaeoidea, Sergestoidea, Caridea, Astacidea, Thalassinidea, Palinura) del Pacífico
}

\author{
Rita Vargas ${ }^{1}$ y Jorge Cortés ${ }^{1,2}$
}

1 Museo de Zoología, Escuela de Biología, Universidad de Costa Rica, San José 2060, Costa Rica, fax (506) 207-4216, Email: ritav@biolgia.ucr.ac.cr

2 Centro de Investigación en Ciencias del Mar y Limnología (CIMAR), Universidad de Costa Rica, San José 2060, Costa Rica.

Recibido 8-IV-1999. Corregido 21-VI-1999. Aceptado 28-VI-1999

\begin{abstract}
A total of 117 species of shrimp and lobster are known from the Pacific coast of Costa Rica. They belong to the Suborders Dendrobranchiata, Superfamily Penaeoidea (26 species) and Pleocyemata, Infraorder Caridea (73), Astacidea (one), Thalassinidea (13), and Palinura (four species). Twenty seven species (23\%) are reported for the first time for Costa Rica. The distribution range of five species is extended, three of which are significant: Pontonia simplex (Holthuis, 1951) from Tenacatitán Bay, México to Culebra Bay, Guanacaste; Veleronia serratifrons (Holthuis, 1951) from La Libertad, Ecuador to Sámara, Guanacaste and Axiopsis serratifrons (A. Milne Edwards, 1873) from Gorgona Island, Colombia to Culebra Bay, Guanacaste. Nine species, two of which are endemic, were described based on specimens collected in Costa Rica. Forty three percent of the tropical eastern Pacific species of shrimps are found on the Pacific coast of Costa Rica.
\end{abstract}

Key words: Shrimps, lobsters, Crustacea, eastern Pacific, Costa Rica, biodiversity.

Debido al reciente interés de conocer o estimar la diversidad marina de Costa Rica se han publicado o se encuentran en preparación una serie de recopilaciones sobre diferentes grupos de invertebrados marinos como esponjas (Cortés 1996), cnidarios (Cortés 19961997), estomatópodos (Vargas y Cortés 1997) camarones y langostas del Caribe (Vargas y Cortés, 1999).

En esta publicación se trata la Superfamilia Penaeoidea del Suborden Dendrobranchiata, y los infraórdenes Caridea, Astacidea, Thalassinidea y Palinura, del suborden Pleocyema- ta. Este tipo de compendios de especies marinas suelen ser de mucha utilidad, como indica Hendrickx (1995a): "las listas para regiones zoogeográficas o provincias facilitan los estudios de biodiversidad en hábitats específicos".

Expediciones realizadas desde el siglo pasado y durante este siglo, por ejemplo, por la U.S. Fish Commission (Albatross), las de la Sociedad Zoológica de Nueva York (Zaca), las de la Fundación Allan Hancock(Velero) o las de la Universidad Nacional Autónoma de México (Puma), han recolectado material de la costa oeste de América, incluyendo Costa Rica. 
Reconocidos taxónomos como L.B. Holthuis y M. K. Wicksten, han descrito especies de carideos de Costa Rica, procedentes de las expediciones anteriormente mencionadas (Vease Holthuis 1951, 1952 y Wicksten 1983). Producto de la expedición de 1993/1994 del R/V Victor Hensen, se publicó una lista de 117 especies de decápodos y estomatópodos recolectados en los golfos de Nicoya y Dulce (Vargas et al. 1996),en donde se mencionan 27 especies de camarones.

La costa pacífica de Costa Rica tiene aproximadamente $1,160 \mathrm{~km}$ y se caracteriza por ser irregular, contando con diferentes tipos de ambientes: estuarios, manglares, fondos lodosos, zonas rocosas, pastos marinos, playas arenosas y arrecifes coralinos. Las mareas tienen una amplitud de hasta $4 \mathrm{~m}$. Por la variedad de hábitats presentes en la costa pacífica de Costa Rica, se espera que la diversidad de los diferentes grupos de invertebrados, en este caso los crustáceos, sea alta.

El objetivo del presente trabajo es dar a conocer 27 nuevos informes de especies para Costa Rica y además hacer un recuento de las especies de camarones y langostas mencionadas en la literatura y las que se encuentran depositadas en el Museo de Zoología de la Escuela de Biología, Universidad de Costa Rica. Esta información nos permitirá tener una idea de la diversidad de estos grupos en aguas del Pacífico costarricense.

\section{MATERIAL Y MÉTODOS}

La mayoría de las especies (72\%) mencionadas en esta publicación se encuentran depositados en la colección de Crustacea del Museo de Zoología, Escuela de Biología, Universidad de Costa Rica. Gran parte del material fue fijado inicialmente con formalina al $10 \%$ y luego almacenado en alcohol etílico al 70\%. Están catalogados bajo las siglas UCR y el número correspondiente. Se siguió la clasificación propuesta para el grupo de los crustáceos por Bowman \& Abele (1982).

Para cada especie se indica: número de especímenes por sexo, o juveniles, localidad y fecha de recolección, y el número de catálogo del Museo de Zoología. Además, se da información sobre su hábitat, si la especie ha sido previamente recolectada en Costa Rica (literatura), su distribución mundial y, en algunos casos, se dan comentarios adicionales. El material fue identificado utilizando las claves respectivas y comparando los especímenes con material de la colección de referencia del Museo de Zoología, la cual contiene numerosas especies cuya identificación ha sido corroborada por diferentes autoridades en el campo, tal como Austin Williams y Rafael Lemaitre (Instituto Smithsonian), Mary Wicksten (Universidad A\&M de Texas). Se utilizan las siguientes abreviaciones: UCR, Universidad de Costa Rica; CR, Costa Rica; s.f. sin fecha; juv., juvenil.

\section{RESULTADOS}

CRUSTACEA Pennant, 1777

DECAPODA Latreille, 1803

DENDROBRANCHIATA Bate, 1888

PENAEOIDEA Rafinesque, 1815

\section{Familia PENAEIDAE Rafinesque, 1815}

Farfantepenaeus brevirostris (Kingsley, 1878)

Material: 20'2\%, playas del Coco, 19.II.1965 (UCR 1325-01). 1 juv., Lepanto, 20.IV.1984 (UCR 1422-01). 1 ơ, golfo de Nicoya, 26.II.1981 (UCR 828-01). 1\%, golfo de Nicoya, 26.II.1981 (UCR 827-01). 20'2\%, golfo de Nicoya, 23.IX.1956 (UCR 40-01). 8G10E, golfo de Nicoya, 10.IV.1980 (UCR 1251-01). $510^{\circ} 42 \%$, golfo de Nicoya, 11.IV.1980 (UCR 781-03). 30 4\%, golfo de Nicoya, 10.XII.1993 (UCR 1986-04). 19, golfo de Nicoya, 11.IV.1980 (UCR 778-01). 19, golfo de Nicoya, 5.III.1981 (UCR 818-02). 19, golfo de Nicoya, 12.IV.1980 (UCR 783-04). 50'7\%, golfo de Nicoya, 11.IV.1980 (UCR 780-02). 30'2\%, frente Hospital, Puntarenas, 10-VII-1980 (UCR 826-01). 50' 4\%, golfo Dulce, 12.XII.1993 (UCR 2008-01). 
Hábitat: sobre fondos arenosos y lodosos, de 20 a 180 m (Hendrickx 1995b).

Informes previos en CR: golfo de Nicoya (Maurer et al. 1984), golfo de Nicoya, bahía Coronado y golfo Dulce (Vargas et al. 1996).

Distribución: de la bahía de San Francisco, California, E.U.A., hasta cabo Blanco, Perú (Méndez 1981).

Comentario: anteriormente Penaeus brevirostris.

Farfantepenaeus californiensis (Holmes, 1900) Material: ninguno

Hábitat: fondos arenosos $\bullet$ lodosos, entre 2 y $180 \mathrm{~m}$, abundante entre 25 y $50 \mathrm{~m}$. Los juveniles se encuentran en estuarios (Hendrickx 1995b).

Informes previos en CR: Costa Rica (Hendrickx 1995b). El informe erroneo para golfo de Nicoya, bahía Coronado y golfo Dulce proporcionado por Vargas et al. (1996), se debió a una mala identificación.

Distribución: de San Francisco, California hasta Callao, Perú e islas Galápagos (Wicksten y Hendrickx 1992).

Comentario: anteriormente Penaeus californiensis.

Metapenaeopsis beebei (Burkenroad, 1938)

Material: ninguno

Hábitat: arena fina (Hendrickx 1996), de 5.5 a $91 \mathrm{~m}$ (Burkenroad 1938).

Informes previos en CR: golfo de Nicoya y Dulce (Vargas et al. 1996), Costa Rica (PérezFarfante y Kensley 1997).

Distribución: de punta San Marcial y Cabo Tepoca, golfo de California, México hasta Panamá (Hendrickx 1996).

Litopenaeus occidentalis (Streets, 1871)

Material: 10", golfo de Nicoya, 13.IV.1980 (UCR 790-02). 10 2\%, golfo de Nicoya, 26.I.1981 (UCR 804-02). 19, golfo de Nicoya, 28.IX.1980 (UCR 797-01). 19, golfo de Nicoya, 25.I.1981 (UCR 807-03). 10'1\%, golfo de Nicoya, 25.I.1981 (UCR 805-04). 10", golfo de Nicoya, 13.IV.1980 (UCR 785-01). 49, golfo de Nicoya, 28.IX.1980 (UCR 794-02). 50' 6\%, golfo de Nicoya, 26.I.1981 (UCR 808-04).
Hábitat: en zonas litorales fangosas, entre 2 y 160 m (Hendrickx 1995b).

Informes previos en CR: golfo de Nicoya (Maurer et al. 1984); playa Cocoroca, golfo de Nicoya (Kitani 1997).

Distribución: del golfo de Tehuantepec, México, hasta isla Lobos de Tierra, Perú e islas Galápagos (Wicksten y Hendrickx 1992).

Comentario: anteriormente Penaeus occidentalis.

Litopenaeus stylirostris (Stimpson, 1874)

Material: $20^{\prime} 29$, oeste y sur de cabo Blanco, 7 8.V.1970 (UCR 1495-01). 90' 109, Lepanto, Puntarenas, 28.X.1984 (UCR 1488-01). 20" 4\%1 juv., Lepanto, Puntarenas, 16.IX.1984 (UCR 2181-01). 1\%, golfo de Nicoya, 14.IV.1980 (UCR 788-02). 19, golfo de Nicoya, 13.IV.1980 (UCR 790-05). 19, golfo de Nicoya, 12.IV.1980 (UCR 784-01). 19, golfo de Nicoya, 12.IV.1980 (UCR 782-02). 19, golfo de Nicoya, 25.I.1981 (UCR 807-02). 10 49, golfo de Nicoya, 25.I.1981 (UCR 805-03). 10', golfo de Nicoya, 29.IX.1980 (UCR 800-02). 29, golfo de Nicoya, 13.IV.1980 (UCR 792-01). 10", golfo de Nicoya, 21.IV.1980 (UCR 829-01). 40" 4\%, golfo de Nicoya, 26.I.1981 (UCR 809-02). 29, golfo de Nicoya, 28.IX.1980 (UCR 794-03). 10", golfo de Nicoya, 26.I.1981 (UCR 808-02). 10", golfo de Nicoya, 16.XII.1993 (UCR 1985-05). $160^{\circ}$, estero Jicaral, río San Pedro, 30.IV.1985 (UCR 1100-01). 5juv., estero Jicaral, 25.VII.1985 (UCR 1206-01). 8juv., estero Jicaral, 26.IX.1985 (UCR 1233-01). 100juv., estero Jicaral, 30.IV.1985 (UCR 1097-01). 70juv., estero Jicaral, 10.V.1985 (UCR 1101-01). 13juv., estero Jicaral, 25.VII.1985 (UCR 1205-01). 15juv., estero Jicaral, 30.VIII.1985 (UCR 122501). 10juv., estero Jicaral, 31.V.1985 (UCR 1156-02). 40juv., estero Jicaral, 30.IV.1985 (UCR 1099-02). 100juv., estero Jicaral, 30.VIII.1985 (UCR 1227-01). 2juv., estero Jicaral, 29.X.1985 (UCR 1239-02). 50juv., estero Jicaral, 30.VIII.1985 (UCR 1226-01). 15juv., estero Jicaral, 31.V.1985 (UCR 1200-01). 10juv., estero Jicaral, 30.IV.1985 (UCR 1095-01). 1ㅇ, de la Angostura hasta Caldera, Puntarenas, 
10.VIII.1980 (UCR 826-02). 10', frente a desembocadura del rio Barranca, golfo de Nicoya, Puntarenas, 16.I.1998 (UCR 2217-02). 20'29, Agujas, Puntarenas, 7.II.1957 (UCR 39-01).

Hábitat: en fondos lodosos o arenosos, entre 5 y 45 m (Hendrichx 1995b).

Informes previos en CR: boca del río Coronado al sureste de Parrita, y mar adentro de Parrita (Pérez-Farfante 1975), golfo de Nicoya (Maurer et al. 1984, Vargas et al. 1996).

Distribución: de punta Abreojos, Baja California, México, hasta Paita, Perú (Wicksten y Hendrickx 1992).

Comentario: anteriormente Penaeus stylirostris.

\section{Litopenaeus vannamei Boone, 1931}

Material: 10", golfo de Nicoya, 23.IV.1981 (UCR 2048-01). 19, golfo de Nicoya (UCR 776-01). 30juv., estero Jicaral, 30.VIII.1985 (UCR 1221-01). 1juv., estero Jicaral, 28.XI.1985 (UCR 1247-02). 1 juv., estero Jicaral, 29.X.1985 (UCR 1240). 8juv., estero Jicaral, 28.II.1985 (UCR 1243-02). 7juv., estero Jicaral, 21.II.1985 (UCR 1248-02). 11juv., estero Jicaral, 31.V.1985 (UCR 1156-01). 20juv., estero Jicaral, 30.VIII.1985 (UCR 1226-02). 15juv., estero Jicaral, 21.VIII.1985 (UCR 1220-02). 8juv., estero Jicaral, 29.X.1985 (UCR 1238-02). 8juv., estero Jicaral, 29.X.1985 (UCR 1235-02). 7juv., estero Jicaral, 26.IX.1985 (UCR 1234-02). 30juv., estero Jicaral, 10.V.1985 (UCR 1101-02). 6juv., estero Jicaral, 31.V.1985 (UCR 120002). 2juv., estero Jicaral, 26.IX.1985 (UCR 1233-02). 20juv., estero Jicaral, 30.VIII.1985 (UCR 1227-02). 5juv., estero Jicaral, 30.IV.1985 (UCR 1093-01). 20'2\%, Dominical, 12.III.1957 (UCR 38-01).

Hábitat: en fondos lodosos, entre 5 y $72 \mathrm{~m}$ (Hendrickx 1996).

Informes previos en CR: mar adentro de $\mathrm{Pa}$ rrita y Dominical (Pérez-Farfante 1975), golfo de Nicoya (Maurer et al. 1984).

Distribución: extremo norte del golfo de California, México, hasta Tumbes, Perú (Wicksten y Hendrickx 1992).

Comentario: anteriormente Penaeus vannamei.
Protrachypene precipua Burkenroad, 1934

Material: 90'3의, golfo de Nicoya, 25.I.1981 (UCR 805-02). 410" 8\%, golfo de Nicoya, 26.I.1981 (UCR 808-01). 2210" 499, golfo de Nicoya, 26.I.1981 (UCR 804-04). 100'2\%, golfo de Nicoya, 29.IX.1 980 (UCR 795-03). 20" 1\%, golfo de Nicoya, 28.IX.1980 (UCR 79602). 4ㅇ, golfo de Nicoya, 29.IX.1980 (UCR 800-05). 10 29, golfo de Nicoya, 25.I.1981 (UCR 812-01). 17\%, golfo de Nicoya, 28.IX.1980 (UCR 794-06). 20'29, golfo de Nicoya, 14-18.XII.1993 (UCR 2010-01). 69, Barranca, Puntarenas, 4.II.1955 (UCR 80-01). Hábitat: en fondos lodosos, a $40 \mathrm{~m}$ y frente a desembocaduras de ríos (Hendrickx 1995b).

Informes previos en CR: golfo de Nicoya (Vargas et al. 1996).

Distribución: del Salvador, hasta Tumbes, Perú (Wicksten y Hendrickx 1992).

Trachysalambria brevisuturae (Burkenroad, 1934) Material: $20^{\circ} 39$, golfo de Nicoya, 14.XII.1993 (UCR 1980-05).

Hábitat: sobre fondos arenosos, capturado entre 4 y 64 m (Hendrickx 1995b).

Informes previos en CR: golfo de Nicoya (Vargas et al. 1996).

Distribución: de banco Arenas y cabo San Lucas, Baja California, México, hasta Tumbes, Perú (Wicksten y Hendrickx 1992).

Comentario: anteriormente Trachypenaeus brevisuturae.

Rimapenaeus byrdi (Burkenroad, 1934)

Material: 20' 2\%, isla Chira, 17.VIII.1955 (UCR 81-01). 1089, golfo de Nicoya, 29.IX.1980 (UCR 799-01). 659, golfo de Nicoya, 28.IX.1980, (UCR 797-03). 320' 21\%, golfo de Nicoya, 26.I.1981 (UCR 806-02). 509, golfo de Nicoya, 29.IX.1980 (UCR 80101). 100 18\%, golfo de Nicoya, 25.I.1981 (UCR 805-05). 1249, golfo de Nicoya, 29.IX.1980 (UCR 795-01). 290'319, golfo de Nicoya, 26.I.1981 (UCR 804-03). 90' 40\%, golfo de Nicoya, 14.IV.1980 (UCR 791-01). $100^{7} 6$ \%, golfo de Nicoya, 25.I.1981 (UCR 807-04). 419, golfo de Nicoya, 29.IX.1980 (UCR 802-02). 690'54\%, golfo de Nicoya, 
13.IV.1980 (UCR 789-01). 10", golfo de Nicoya, 13.IV.1980 (UCR 965-01). 20"4\%, golfo de Nicoya, 16.II.1994 (UCR 2011-02). 70' 129, golfo de Nicoya, 26.II.1981 (UCR 817-01). $250^{7} 22$, golfo de Nicoya, 14.IV.1980 (UCR 788-03). 469, golfo de Nicoya, 29.IX.1980 (UCR 816-01). 60" 23\%, golfo de Nicoya, 13.IV.1980 (UCR 792-02). 50" 129, golfo de Nicoya, 26.I.1981 (UCR 809-01). 50"69, golfo de Nicoya, 26.I.1981 (UCR 808-03). 869, golfo de Nicoya, 29.IX.1980 (UCR 800-04). 779, golfo de Nicoya, 29.IX.1980 (UCR 79801). 19, golfo Dulce, boca Guarumal, 26.II.1992 (UCR 1812-01).

Hábitat: ambientes marinos y estuarinos, en fondos lodosos de la plataforma continental, entre 2 y 40 m (Hendrickx 1995b).

Informes previos en CR: golfo de Nicoya (Maurer et al. 1984; Vargas et al. 1996).

Distribución: de México, hasta Paita, Perú (Wicksten y Hendrickx 1992).

Comentario: anteriormente Trachypenaeus byrdi.

\section{Rimapenaeus faoe (Obarrio, 1954)}

Material: $10^{\circ}, 2 \mathrm{~km}$ afuera de isla Negritos, VII.1980 (UCR 831-01). 10'89, golfo de Nicoya, 16.XII.1993 (UCR 1989-02). 29, golfo de Nicoya, 14.IV.1980 (UCR 787-01). 99, golfo de Nicoya, 28.IX.1980 (UCR 794-04). 69, golfo de Nicoya, 28.IX.1980 (UCR 797-02). 22\%, golfo de Nicoya, 28.IX.1980 (UCR 79603). $10^{7} 4$ \%, golfo de Nicoya, 25.I.1981 (UCR 810-01). 199, golfo de Nicoya, 28.IX.1980 (UCR 802-01). 3\%, golfo de Nicoya, 10.IV.1980 (UCR 775-01). 69, golfo de Nicoya, 29.IX.1980 (UCR 800-01). 50"3\%, golfo de Nicoya, 12.IV.1980 (UCR 784-02). 50" 5\%, golfo de Nicoya, 25.I.1981 (UCR 807-01). 20\%, golfo de Nicoya, 26.I.1981 (UCR 80903). 40'69, golfo de Nicoya, 14.II.1994 (UCR 2011-03).

Hábitat: capturada hasta $24 \mathrm{~m}$ de profundidad (Hendrickx 1995b).

Informes previos en CR: golfo de Nicoya (Maurer et al. 1984), golfo de Nicoya y golfo Dulce (Vargas et al. 1996).
Distribución: desde isla Altamura, Sinaloa, México, hasta el golfo de Guayaquil, Ecuador (Wicksten y Hendrickx 1992).

Comentario: anteriormente Trachypenaeus faoe.

Rimapenaeus fuscina (Pérez-Farfante, 1971) Informes previos en CR: el informe para golfo de Nicoya, bahía Coronado y golfo Dulce dado por Vargas et al. (1996) se debió a una mala identificación.

Comentario: anteriormente Trachypenaeus fuscina.

Rimapenaeus pacificus (Burkenroad, 1934)

Material: 69\%, golfo de Nicoya, 10.IV.1980 (UCR 779-01). 160", golfo de Nicoya, 10.IV. 1980 (UCR 779-03). 19, golfo de Nicoya, 1980 (UCR 967-01). 1\%, Parque Nacional Manuel Antonio, Puntarenas, 21.XI.1996 (UCR 2182-04). 10 4\%, Sierpe, Puntarenas, 13.XII.1993 (UCR 1990-02). 20² 2\%, Golfo Dulce, 12.XII.1993 (UCR 2008-02).

Hábitat: en fondos blandos, entre 2 y $100 \mathrm{~m}$, en la plataforma continental (Hendrickx 1995b).

Informes previos en $\mathbf{C R}$ : golfo de Nicoya (Maurer et al. 1984), golfo de Nicoya y bahía Coronado (Vargas et al. 1996).

Distribución: desde cabo San Quintín, Baja California, México, hasta Huacho, Perú (Wicksten y Hendrickx 1992).

Comentario: anteriormente Trachypenaeus pacificus.

Xiphopenaeus kroyeri (Heller, 1862)

Material: $80^{\circ} 10$, golfo de Nicoya, 26.I.1981 (UCR 804-01). 32\%, golfo de Nicoya, 28.IX.1980 (UCR 796-01). 40'2\%, golfo de Nicoya, 25.I.1981 (UCR 805-01). 10"1 1\%, golfo de Nicoya, 10.IV.1980 (UCR 777-01). 119, golfo de Nicoya, 29.IX.1980 (UCR 795-02). 19, golfo de Nicoya, 12.IV.1980 (UCR 784-03). 20" 5 , golfo de Nicoya, 15.XII.1993 (UCR 199605). $160^{\prime \prime} 139$, golfo de Nicoya, 26.I.1981 (UCR 806-01). 419, golfo de Nicoya, 28.IX.1980 (UCR 794-05). 80 149, golfo de 
Nicoya, 10.IV.1980 (UCR 776-03). 130'37\%, golfo de Nicoya, 26.II.1981 (UCR 817-02). 669, golfo de Nicoya, 28.IX.1980 (UCR 797-04). $540^{7} 48$, golfo de Nicoya, 25.I.1981 (UCR 80705). 179, golfo de Nicoya, 28.IX.1980 (UCR 802-03). 3\%, de la Angostura a Caldera, Puntarenas, 10.VII.1980 (UCR 826-03). 30' 3\%, Barranca, Puntarenas, 7.VI.1954 (UCR 42-01). 80" 13 , frente a la desembocadura del río Barranca, golfo de Nicoya, 16.I.1998 (UCR 2217-01).

Hábitat: en aguas poco profundas, frente a desembocaduras de los ríos hasta $70 \mathrm{~m}$ de profundidad, en fondos blandos, limosos o arcillosos (Hendrickx 1995b).

Informes previos en CR: golfo de Nicoya (Maurer et al. 1984), golfo de Nicoya y golfo Dulce (Vargas et al. 1996).

Distribución: afuera de punta Piaxtla, Sinaloa, México, hasta Paita, Perú. De Carolina del Norte hasta el golfo de México, del Mar Caribe hasta Santa Catarina, Brasil (Pérez-Farfante y Kensley 1997).

Comentario: anteriormente Xiphopenaeus riveti Bouvier, 1907, hasta que fue establecido como sinónimo de $X$. kroyeri por Pérez-Farfante y Kensley (1997).

\section{Familia SOLENOCERIDAE Wood-Mason} and Alcock, 1891

Haliporoides diomedeae (Faxon, 1893)

Material: $10^{\prime} 1 \%$, costa pacífica, s.f. (UCR 1807-07).

Hábitat: fondos lodosos entre 240 y $1,865 \mathrm{~m}$ (Hendrickx 1995b).

Informes previos en CR: es primer informe para Costa Rica.

Distribución: de Panamá al sur hasta Chile (Pérez-Farfante y Kensley 1997).

Comentario: se amplia distribución al norte hasta la costa pacífica de Costa Rica.

\section{Hymenopeaneus doris (Faxon, 1893)}

Material: ninguno.

Hábitat: se ha recolectado de fondos entre 549 a 4,802 m (Méndez 1981). Burkenroad (1938) lo menciona como una especie de hábitos semi-bentónicos.
Informes previos en CR: Costa Rica, $10^{\circ} 14^{\prime} \mathrm{N}$ (Faxon 1895), Costa Rica (Pérez-Farfante 1977).

Distribución: desde punta Chivoto, golfo de California, México, hasta las afueras de Guañape, Perú (Wicksten y Hendrickx 1992).

Comentario: localidad tipo cabo Velas, Costa Rica (Hendrickx y Estrada Navarrete 1996).

Hymenopenaeus nereus (Faxon, 1893)

Material: ninguno.

Hábitat: en fondos entre 1,300 y 3,300 m (Méndez 1981).

Informes previos en CR: isla del Coco (Faxon 1895), al sur de cabo Blanco $5^{\circ} 30^{\prime} \mathrm{N}$, $86^{\circ} 45^{\prime} \mathrm{W}$ (Pérez-Farfante 1977).

Distribución: de las afueras de cabo Blanco, Costa Rica, hasta el norte de punta Galena, Ecuador e islas Galápagos (Méndez 1981).

\section{Solenocera agassizii Faxon, 1893}

Material: $420^{\prime} 15 \%$, golfo de Nicoya, (UCR 778-04). $160^{\circ} 3$ 39, golfo de Nicoya, 10.IV.1980 (UCR 779-05). 100'2\%, golfo de Nicoya, 11.IV.1980 (UCR 780-01). 50'7\%, golfo Dulce, 15.XII.1993 (UCR 2007-01).

Hábitat: sobre fondos lodosos y arenosos, entre 16 y $384 \mathrm{~m}$ de profundidad (Hendrickx 1995b).

Informes previos en CR: mar adentro de cabo Blanco (Pérez-Farfante 1988).

Distribución: mar adentro de cabo Blanco, Costa Rica, hasta islas Lobos de afuera, Perú (Wicksten y Hendrickx 1992)

Solenocera florea Burkenroad, 1938

Material: $40^{7} 6 \%$, golfo de Nicoya, 1981 (UCR 2047-01). 10', Parque Marino Ballena, 14.XII.1993 (UCR 1987-06). 50'7\%, Parque Marino Ballena, Puntarenas, 14.XII.1993 (UCR 1980-09). 1\%, frente a peña Bruja, Parque Nacional Santa Rosa, Guanacaste, 2.VII.1980 (UCR 832-04).

Hábitat: en fondos de arena fina, entre 13 y $183 \mathrm{~m}$ de profundidad (Hendrickx 1995b).

Informes previos en CR: golfo de Nicoya (Maurer et al. 1984), golfos de Nicoya y Dulce (Vargas et al. 1996). 
Distribución: bahía Todos los Santos, Baja California, México, hasta el suroeste de Pimental, Perú (Wicksten y Hendrickx 1992).

Solenocera mutatorBurkenroad, 1938

Material: $60^{\circ} 10 \%$, golfo de Nicoya, 16.XII.1993 (UCR 1986-03). 160'18\%, golfo Dulce, 12.XII.1993 (UCR 2105-01). 19, Parque Marino Ballena, 26.I.1994 (UCR 2185-01). 19, golfo Dulce, 19.I.1994 (UCR 2174-01).

Hábitat: fondo de tipo lodoso, principalmente desde $90 \mathrm{~m}$ de profundidad en adelante (Hendrickx 1995b).

Informes previos en CR: golfo de Nicoya, bahía Coronado y golfo Dulce (Vargas ett al. 1996).

Distribución: mar afuera de punta Santa Bárbara, California, E.U.A., hasta isla Lobos de Tierra, Perú (Wicksten y Hendrickx 1992).

Familia SICYONIIDAE Ortmann, 1898

\section{Sicyonia affinis Faxon, 1893}

Material: ninguno

Hábitat: sobre fondos rocosos y de cascajo entre 77 y 205 m (Hendrickx 1995b).

Informes previos en CR: isla del Coco (Faxon 1893).

Distribución: del golfo de California a Paita, Perú; isla del Coco e isla Malpelo (Wicksten y Hendrickx 1992).

Comentario: la localidad tipo es la isla del Coco (Faxon 1893, Hendrickx 1984).

Sicyonia aliaffinis(Burkenroad, 1934)

Material: 1 \%, $2 \mathrm{~km}$ al sur de islas Catalinas, Guanacaste, 2.XI.1983 (UCR 1401-01).

Hábitat: se ha capturado de fondos muy diversos pero predominan en fondos suaves desde arena fina a limo, desde $4 \mathrm{~m}$ hasta 242 $\mathrm{m}$, pero es más frecuente en el intervalo de 25 a $90 \mathrm{~m}$ de profundidad (Hendrickx 1995b).

Informes previos en $\mathbf{C R}$ : es primer informe para Costa Rica.

Distribución: de Baja California a Perú (Hendrickx 1984).
Sicyonia disdorsalis (Burkenroad, 1934)

Material: $40^{\circ} 9 \%$, frente peña Bruja, Parque Nacional Santa Rosa, 2.VII.1980 (UCR 83203). 1\%, golfo de Nicoya, 25.I.1981 (UCR 812-02). 20'4\%, golfo de Nicoya, 12.IV.1980 (UCR 783-03). 129, golfo de Nicoya, 16.XII.1993 (UCR 1989-03). 10 39, golfo de Nicoya, 12.IV.1980 (UCR 782-03). 140' 22\%, golfo de Nicoya, 10.IV.1980 (UCR 779-04). $10^{7}$ 39, Parque Marino Ballena, Puntarenas, 24.XI.1996 (UCR 2172-04).

Hábitat: se encuentra en arena fina, muy fina y limo, a profundidades entre 3 y $100 \mathrm{~m}$ (Hendrickx 1984).

Informes previos en CR: cabo B lanco (Hendrickx y Estrada Navarrete 1996), golfo de Nicoya (Maurer et al. 1984), golfo de Nicoya, bahía Coronado y golfo Dulce (Vargas et al. 1996).

Distribución: de la costa central de Sonora y el golfo de California, México, a Pisco, Perú (Wicksten y Hendrickx 1992).

Sicyonia disedwardsi (Burkenroad, 1934)

Material: 1 \%, playa Mostrencal, bahía Junquillal, 13.III.1996 (UCR 2149-01). 10', cabo Blanco, 28.IV.1973 (UCR 2184-01). 20", isla del Caño, 24.XI.1996 (UCR 2183-03). 10' 5\%, golfo Dulce, 12.XII.1993 (UCR 1995-01).

Hábitat: sobre fondos duros de arena gruesa y fragmentos y valvas de conchas, algunas veces mezclados con pequeñas piedras o fragmentos de coral (Hendrickx 1984).

Informes previos en CR: bahía Culebra, cabo Blanco, golfo Dulce e isla Manuelita, isla del Coco (Hendrickx y Estrada-Navarrete 1996); golfos de Nicoya y Dulce (Vargas et al. 1996).

Distribución: de isla Santa María, Baja California y el golfo de California, México, a punta Caracoles, Darién, Panamá (Wicksten y Hendrickx 1992).

\section{Sicyonia laevigata Stimpson, 1871}

Material: 7 juv, Santa Elena, Guanacaste, 14.X.1970 (UCR 968-07). 19, isla San José, islas Murciélago, 13.IV.1970 (UCR 970-01). Hábitat: prefiere fondos conchosos y bahías 
someras protegidas, no más allá de los $90 \mathrm{~m}$ de profundidad (Hendrickx 1984).

Informes previos en CR: primer informe para Costa Rica.

Distribución: de Mazatlán, Sinaloa, México, hasta punta Paitilla, Panamá en la costa pacífica. En el Atlántico de cabo Hatteras, Carolina del Norte, E.U.A., hasta Anse de Zimbros, Santa Catarina, Brasil, incluyendo las Antillas (Wicksten y Hendrickx 1992).

Sicyonia martini Pérez-Farfante \& Boothe, 1981 Material: $1 \sigma^{7} 1$ \&, isla David, bahía Junquillal, Guanacaste 12.III.1996 (UCR 2151-01). 20" 7 , frente a peña Bruja, Parque Nacional Santa Rosa, 2.VII.1980 (UCR 832-02).

Hábitat: sobre arena, rocas, lodo y escombros coralinos, de 14 a 32 m (Hendrickx 1984).

Informes previos en CR: punta Quepos, golfo Dulce (Pérez-Farfante 1985).

Distribución: isla Santa Margarita, México; golfo de California al sur de isla Tiburón, Sonora, México, hasta el suroeste de punta Ana María, Panamá (Wicksten y Hendrickx 1992).

Sicyonia picta Faxon, 1893

Material: $10^{7} 1 \%$, frente a peña Bruja, Parque Nacional Santa Rosa, 2.VII.1980 (UCR 83201). $100^{\circ} 40$, golfo de Nicoya, 11.IV.1980 (UCR 778-05). 90' 25\%, golfo de Nicoya, 15.XII.1993 (UCR 1982-03). 90' 25\%, golfo de Nicoya, 12.IV.1980 (UCR 782-01). 80' 31우, golfo de Nicoya, 11.IV.1980 (UCR 780-03). $10^{7} 1$ \%, golfo de Nicoya, 24.IV.1981 (UCR 833-01). 80' 67\%, entre cabo Blanco y punta Herradura, 19-21.V.1970 (UCR 244-01). 30’ 5우, golfo Dulce, 11.XII.1993 (UCR 2005-04).

Hábitat: habita sustratos que van desde arena fina o gruesa hasta fragmentos de concha sobre arena gruesa, del nivel de la playa hasta $400 \mathrm{~m}$ de profundidad (Hendrickx 1984).

Informes previos en CR: cabo Blanco (PérezFarfante 1985), golfo de Nicoya (Maurer et al. 1984), golfo de Nicoya, bahía Coronado y golfo Dulce (Vargas et al. 1996).

Distribución: desde bahía Magdalena, Baja California, México, hasta la isla Lobos de Afuera, Perú (Wicksten y Hendrickx 1992).
SERGESTOIDEA Dana, 1852

Familia SERGESTIDAE Dana, 1852

Sergestes pestafer Burkenroad, 1937

Material: ninguno.

Hábitat: en la columna de agua entre 180$250 \mathrm{~m}$ (Hendrickx y Estrada-Navarrete 1996).

Informes previos en $\mathbf{C R}$ : cerca de la isla del Coco (Hendrickx y Estrada-Navarrete 1996).

Distribución: Pacífico oeste, de Baja California a islas Galápagos (Hendrickx y EstradaNavarrete 1996).

\section{PLEOCYEMATA Burkenroad, 1963 CARIDEA Dana, 1852}

\section{Familia OPLOPHORIDAE Dana,1852}

Acanthephyra curvirostris Wood-Mason, 1891 Material: 19 , costa pacífica, s.f. (UCR 180709).

Hábitat: recolectada en arrastres de media agua y a profundidades entre $300-4970 \mathrm{~m}$ (Hendrickx y Estrada-Navarrete 1996).

Informes previos en $\mathbf{C R}$; es primer informe para Costa Rica.

Distribución: de la isla Vancouver, a Perú incluyendo el golfo de California; Oceános IndoPacífico y Atlántico (Hendrickx y Estrada-Navarrete 1996).

Meningodora mollis Smith, 1882

Material: ninguno.

Hábitat: en arrastre vertical de $2000 \mathrm{~m}$ a la superficie (Hendrickx y Estrada-Navarrete 1996).

Informes previos en $\mathbf{C R}$ : al sureste de la isla del Coco (Faxon 1895)

Distribución: de Oregon, E.U.A., hasta Panamá e islas Galápagos; Oceános Indico y Atlántico (Hendrickx y Estrada-Navarrete 1996).

Comentario: fue informada para la isla del Coco como Notostomus fragilis por Faxon (1893). 


\section{Familia PASIPHAEIDAE Dana, 1852}

\section{Leptochela serratorbita Bate, 1888}

Material: ninguno.

Hábitat: fondos arenosos o lodosos, desde la superficie a $110 \mathrm{~m}$, se puede atraer de noche con luz (Wicksten 1983).

Informes previos en CR: bahía Santa Elena (puerto Parker) (Wicksten 1983).

Distribución: en el Atlántico desde Carolina del Norte, E.U.A., a bahía Campeche, México, e isla Antigua. En el Pacífico de isla Angel de la Guarda, golfo de California, México, a bahía Santa Elena (puerto Parker), Costa Rica (Wicksten 1983).

\section{Pasiphaea magna Faxon, 1893}

Material: 2\%, costa pacífica, s.f. (UCR 180706).

Hábitat: batipelágica pero ha sido capturada a media agua (Hendrickx y Estrada-Navarrete 1996).

Informes previos en CR: es primer informe para Costa Rica.

Distribución: de Oregón, E.U.A., incluyendo el sureste del golfo de California y golfo de $\mathrm{Pa}$ namá hasta la costa de Perú (Hendrickx y Estrada-Navarrete 1996).

\section{Familia GNATHOPHYLLIDAE Dana, 1852}

Gnathophyllum panamense Faxon, 1893

Material: 10", puesto playita, isla del Caño, 26.V.1989 (UCR 1914-07). 10', isla del Caño, 21.III. 1970 (UCR 478-01). 10", isla del Caño, 21.III.1970 (UCR 477-01). $50^{\circ 3}$ \%, lado N.E. bahía Wafer, isla del Coco, 27.III.1978 (UCR 1673-15).

Hábitat: entre rocas, de la orilla a $20 \mathrm{~m}$ (Wicksten 1983).

Informes previos en CR: es primer informe para Costa Rica.

Distribución: de Cabeza de Ballena, Baja California Sur, México y el golfo de California a islas Galápagos (Wicksten 1983).

\section{Familia PALAEMONIDAE Rafinesque, 1815}

Brachycarpus biunguiculatus (Lucas, 1849) Material: $40^{\circ} 4$, lado Norte de isla San José, islas Murciélago, 7.XII.1968 (UCR 28901). 10̛ 1ㅇ, isla del Caño, 25.II.1970 (UCR 290-01). 10 1\%, isla del Caño, 12.IV.1970 (UCR 291-01). 3o, isla del Caño, 6.IV.1970 (UCR 570-01). 10", isla del Caño, 5.IV.1970 (UCR 571-01). 10'19, bajo Alcyone, isla del Coco, 2.IV.1992 (UCR 1760-01). 60' 119, bahía Waffer, isla del Coco, 27.III.1978 (UCR 1673-09). 10", isla del Coco, 1-9.IV.1992 (UCR 1734-01). 39, isla Manuelita, isla del Coco, 7.IV.1992 (UCR 1771-01). 10", isla del Coco, 5.IV.1992 (UCR 1740-01). 10", isla del Coco, 7.IV.1992 (UCR 1742-01). 10", isla del Coco, 4.IV.1992 (UCR 1780-01). 10' 2\%, isla del Coco, 3.IV.1992 (UCR 1781-01).

Hábitat: principalmente en aguas someras entre coral (Holthuis 1952).

Informes previos en CR: bahía Santa Elena, playa Blanca e isla del Coco (Holthuis 1952).

Distribución: Pantropical (Abele y Kim 1986).

Fennera chacei Holthuis, 1951

Material: ninguno

Hábitat: epizoótico sobre corales (Holthuis 1951)

Informes previos en CR: bahía Santa Elena, Guanacaste (Holthuis 1951).

Distribución: de la isla Isabel, Sinaloa, México, hasta Colombia e islas Galápagos. En el Pácifico Indo-oeste, del Océano Indico a Australia y Hawai'i (Wicksten y Hendrickx 1992).

Harpiliopsis depressa (Stimpson, 1860)

Material: $40^{\circ} 4$ \%, Tamarindo, Guanacaste, 27.I.1967 (UCR 293-01). 10 191juv., isla del Caño, 23.II.1970 (UCR 402-01).

Hábitat: aguas someras entre corales (Wicksten 1983).

Informes previos en CR: bahía Wafer, isla del Coco (Wicksten 1983). 
Distribución: del golfo de California, México, hasta la isla Gorgona, Colombia, islas Galápagos e isla del Coco (Wicksten 1983). También del Mar Rojo a la Polinesia (Wicksten y Hendrickx 1992).

\section{Macrobrachium americanum Bate, 1868a}

Material: $40^{7} 3$ 3 , estero en boca río Copey, Jacó, 10.I.1967 (UCR 1118). 30² 2\%, boca río Copey, Jacó, 10.I.1967 (UCR 278-01). 50" 169, isla del Caño, 24.II.1970 (UCR 296-01). $10^{7} 1$ juv., isla del Caño, III.1970 (UCR 96901). 40", isla del Caño, 20.II.1970 (UCR 132101). 20", Sierpe, 27.II.1985 (UCR 1152-01). $330^{\circ} 139$, playa Kativo, Osa, 14.XII.1979 (UCR 1143-03). 140'1\%, boca río Esquinas, 14.XII.1979 (UCR 1144-01). 10', boca Guarumal, Térraba-Sierpe, 26.XI.1992 (UCR 193603). 1\%, estero frente a la salida principal de Golfito, 4.I.1967 (UCR 276-01). 10', isla del Coco, s.f. (UCR 1399-01). 30'5\%, isla del Coco, 4.IV.1992 (UCR 1579-01). 30'2\%, isla del Coco, 4.IV.1992 (UCR 1568-01).

Hábitat: agua dulce (Holthuis 1952). Ambientes salobres en esteros y desembocadura de ríos (esta publicación).

Informes previos en CR: bahías Chatham y Wafer, isla del Coco; golfo Dulce (Holthuis 1952). A lo largo de la costa Pacífica (Cedeño 1986).

Distribución: Guaymas, Sonora; cabos La Paz y San Lucas, Baja California Sur, México, a río Chira, Perú, islas Galápagos e isla del Coco (Wicksten y Hendrickx 1992).

Comentario: solo se incluyó el material procedente de ambientes salobres.

\section{Macrobrachium panamense Rathbun, 1912}

Material: $20^{\circ} 19$, Mata de Limón, 8.V.1966 (UCR 552-01). 19, estero Jicaral, 30.VIII.1985 (UCR 1222-04). 19, estero Jicaral, 30.VIII.1985 (UCR 1226-06). 30'6\%, estero Jicaral, 28.XI.1985 (UCR 1246-04). 10'2\%, Boca Guarumal, golfo Dulce, 26.XI.1992 (UCR 1936-01). Hábitat: agua dulce y en las orillas de la costa (Holthuis 1952).

Informes previos en CR: ríos Bebedero y Tenorio, Guanacaste (Pesta 1931); bahía Culebra (Holthuis 1952).
Distribución: del Golfo de Fonseca, Honduras, hasta Perú (Méndez 1981).

Macrobrachium tenellum (Smith, 1871)

Material: $10^{\top} 2 \%$, boca río Guarumal, golfo Dulce, 26.XI.1992 (UCR 1936-02).

Hábitat: orillas de esteros y agua dulce (Holthuis 1952). En agua dulce y salobre, fondos arenosos, lodosos o rocosos. En aguas con salinidad del $20 \%$. Adultos y subadultos con frecuencia en praderas de Ruppia o cerca de la boca de lagunas salobres (Hendrickx 1995b).

Informes previos en CR: bahía Culebra (puerto Culebra) (Holthuis 1952).

Distribución: de Baja California, México, al norte de Perú (Holthuis 1952).

Palaemon gracilis (Smith, 1871)

Material: 2 Boca Guarumal, desembocadura del río Térraba-Sierpe, 26.XI.1992 (UCR 1916-02).

Hábitat: lagunas costeras y estuarios de baja salinidad (hasta $8 \%$ ) y en agua dulce (Hendrickx 1995b).

Informes previos en CR: río Lagarto, boca del río Esquinas y quebrada Suira, río Esquinas, golfo Dulce (Cedeño 1986).

Distribución: de Sinaloa, México, a Panamá (Wicksten 1983).

Palaemon ritteri Holmes, 1895

Material: 4\%, Tamarindo, 27.I.1967 (UCR 294-01). 950' 2169, Jicaral, Puntarenas, 28.XI.1985 (UCR 1246-05). 60" 1\%, playa Escondida, Herradura, 8.XI.1995 (UCR 219201). 50" 19, Jacó, 11.I.1967 (UCR 548-01). $20^{\circ}$ 1\%, Jacó, 9.IV.1966 (UCR 549-01). 30', punta Mala, Puntarenas, 17.I.1969 (UCR 563-01). 220'43\%, isla del Coco, 27.III.1978 (UCR 1673-10). 590' 1199, isla del Coco, 7.II.1993 (UCR 2060-01).

Hábitat: a lo largo de la costa en rocas, arena y arrecifes (Holthuis 1952). En aguas someras, pozos de mareas y zona de mareas (Méndez 1981). En la línea de la playa y hasta $38 \mathrm{~m}$, se congrega por deba jo de las rocas más grandes. Es una especie limpiadora facultativa, devorando parásitos 
de otras especies sin especificidad de huésped (Hendrickx 1995b).

Informes previos en CR: bahía Wafer, isla del Coco (Holthuis 1952).

Distribución: San Diego, California, E.U.A.; costa exterior de Baja California; el golfo de California; la costa oeste de México, isla del Coco, Panamá, Colombia, Ecuador e islas Galápagos (Wicksten 1983).

\section{Palaemonella holmesi (Nobili 1907)}

\section{Material: ninguno.}

Hábitat: vive en fondos arenosos o rocosos; sólo una vez se ha informado de fondos lodosos. Probablemente prefiere vivir entre corales y pastos marinos (Holthuis 1951). De 2 a $90 \mathrm{~m}$ (Wicksten 1983).

Informes previos en CR: bahía Culebra y mar afuera al sur de islas Viradores (Holthuis 1951) Distribución: de California a Ecuador (Holthuis 1951, Wicksten 1983).

\section{Palaemonetes schmitti Holthuis, 1950}

Material: $120^{\circ} 16 \%$, desembocadura del río San Pedro, Jicaral, 28.XI.1985 (UCR 124303). $180^{\circ} 79$, desembocadura del río San Pedro, Jicaral, 10.V.1985 (UCR 1101-05). 70 5\%, desembocadura del río San Pedro, Jicaral, 10.V.1985 (UCR 1101-03).

Hábitat: agua dulce y salobre (Holthuis 1952, Abele y Kim, 1989).

Informes previos en CR: golfo de Nicoya (Wehrtmann 1990).

Distribución: del golfo de Nicoya, Costa Rica, a playa Venado, zona del Canal, este de la ciudad de Panamá, playa San Francisco y Miraflores, Panamá (Wehrtmann 1990).

Periclimenaeus spinosus Holthuis, 1951

Material: ninguno.

Hábitat: aguas someras en coral (Holthuis 1951).

Informes previos en CR: cerca del sur de islas Viradores, bahía Culebra (Holthuis 1951).

Distribución: de bahía Concepción, golfo de California, hasta el sur de islas Viradores, bahía Culebra, Costa Rica (Ríos 1986).
Comentarios: el holotipo procede de sur de islas Viradores, bahía Culebra, Guanacaste (Holthuis 1951).

Periclimenes infraspinis (Rathbun, 1902)

Material: $30^{\circ} 1$ juv., Islotes, golfo Dulce, 12.IV.1997 (UCR-2243-01). 30" 2\%, Islotes, golfo Dulce, 12.IV.1997 (UCR-2243-02). 10', Islotes, golfo Dulce, 12.IV.1997 (UCR-2243-03).

Hábitat: vive en aguas someras, cerca de la costa, en fondos arenosos o lodosos entre hierbas (Holthuis 1951)

Informes previos en $\mathbf{C R}$ : bahía Salinas y bahía Santa Elena, Guanacaste (Holthuis 1951)

Distribución: desde San Diego, California, hasta Costa Rica (Holthuis 1951).

\section{Periclimenes lucasi Chace 1937}

Material: ninguno.

Hábitat: aguas litorales someras en fondos arenosos y lodosos (Holthuis 1951). De la línea inferior de marea hasta $90 \mathrm{~m}$, ocasionalmente asociado con ceriántidos (Wicksten 1983).

Informes previos en CR: playa Blanca y bahía Culebra, Guanacaste (Holthuis 1951).

Distribución: de Baja California, México, hasta el sur de Panamá (Holthuis 1951, Wicksten 1983, Wicksten y Hendrickx 1992).

Pontonia margarita Smith, 1869

Material: 10 1\%, bahía Salinas, 28.VII.1980 (UCR 1572-01). 10 1\%, islas Murciélago, 12 13.VIII.1990 (UCR 1685-01). 10 19, islas Murciélago, golfo Papagayo, 12-13.VIII.1990 (UCR 1967-01). 10 19, isla Colorada, Guanacaste, 5.III.1994 (UCR 1958-01). 10'1\%, playa Iguanitas, bahía Culebra, Guanacaste, 19.XI.1994 (UCR 1999-01). 19, Conchal, Guanacaste, 6-9.V.1982 (UCR 1733-01). 130" 179, Conchal, Guanacaste, VII.1976 (UCR 1682-01). 30'3요, playa Hermosa, Guanacaste, 28.III.1997 (UCR 2204-01). 10' 1\%, playa Escondida, Herradura, 5.I.1994 (UCR 2199-01). Hábitat: comensal en la ostra perlera Pinctada mazatlanica y especies relacionadas, en aguas someras (Wicksten 1983). En el presente trabajo se informa la presencia de la especie en el bivalvo Modiolus capax (Conrad, 1837). 
Informes previos en CR: bahía Santa Elena, playa Blanca y bahía Culebra, Guanacaste (Holthuis 1951); isla Olacinta, Puntarenas (Wicksten 1983); playa Panamá, bahía Culebra (Cabrera y Solano 1996).

Distribución: costas este y oeste de Florida en el Atlántico, del golfo de California, México, a Colombia, e islas Galápagos en el Pacífico (Wicksten y Hendrickx 1992).

\section{Pontonia pinnae Lockington, 1878}

Material: ninguno.

Hábitat: en la cavidad del manto de Pinna rugosa.

Informes previos en CR: Puntarenas, Costa Rica (Boone 1931).

Distribución: de bahía Chollas, golfo de California, a islas Perlas, Panamá (Holthuis 1951, Wicksten 1983).

Pontonia simplex Holthuis, 1951

Material: $10^{\circ} 2 \%$, bahía Santa Elena, 21.II.1965 (UCR 444-01). 160 20\%, playa Iguanitas, bahía Culebra, Guanacaste, 4.XII.1995 (UCR 2202-01).

Hábitat: en la orilla asociada con Pinna sp. (Wicksten 1983). Comensal de Pinna rugosa (esta publicación).

Informes previos en $\mathbf{C R}$ : es primer informe para Costa Rica.

Distribución: de puerto Escondido, golfo de California; bahía de Tenacatitlan, México (Wicksten 1983).

Comentario: se amplia la distribución a bahía Culebra, Guanacaste, Costa Rica.

Pontonia spighti Fujino, 1972

Material: ninguno.

Hábitat: asociado al ascidio Rhopalaea birkelandi, en aguas someras de la zona sublitoral (Fujino 1972).

Informes previos en CR: playas del Coco, Guanacaste, Costa Rica (Fujino 1972).

Distribución: conocido solamente de la localidad previamente citada (Wicksten y Hendrickx 1992).

Comentario: especie endémica, sólo ha sido recolectada en la localidad tipo.
Veleronia laevifrons Holthuis, 1951

Material: $20^{\circ} 2 \%$, islas Palmitas, Guanacaste 27.VIII.1997 (UCR 2243-02). 2072 29, Sámara, Guanacaste, 28.V.1997 (UCR-2233-02).

Hábitat: recolectado con rocas y arena, de 3 a $13 \mathrm{~m}$ (Holthuis 1951). Asociado con octocorales del género Leptogorgia (esta publicación)

Informes previos en CR: es primer informe para Costa Rica.

Distribución: bahías de San Carlos y Guaymas, Sonora, golfo de California; mar afuera de cabo San Francisco, Ecuador e islas Galápagos (Wicksten y Hendrickx 1992).

Veleronia serratifrons Holthuis, 1951

Material: 19, Sámara, Guanacaste, 28.V.1997 (UCR-2233-01).

Hábitat: recolectado con rocas y conchas de 7$18 \mathrm{~m}$ (Holthuis 1951). Asociado con octocorales del género Leptogorgia (esta publicación).

Informes previos en CR: es primer informe para Costa Rica.

Distribución: mar afuera de La Libertad, Ecuador (Wicksten y Hendrickx 1992).

Comentario: se amplia la distribución al norte a Sámara, Guanacaste, Costa Rica.

\section{Waldola schmitti Holthuis, 1951}

Material: $50^{\prime \prime} 14 \%$, isla San Pedrito, islas Murciélago, Guanacaste, 26.V.1996 (UCR-2247-02).

Hábitat: recolectado con arena, nuliporos, rocas, gorgonidos y conchas (Holthuis 1951). Asociado con el coral negro Antipathes panamensis Verrill, 1869 (esta publicación).

Informes previos en CR: es primer informe para Costa Rica.

Distribución: de mar afuera de isla Isabel, Nayarit, México, a mar afuera de islas Gorgona, Colombia (Wicksten y Hendrickx 1992).

Comentario: se amplia la distribución al norte a Sámara, Guanacaste, Costa Rica.

Familia ALPHEIDAE Rafinesque, 1815

Alpheus aequus Kim \& Abele, 1988

Material: ninguno.

Hábitat: playa de esquisto, de la orilla hasta 5 m de profundidad (Kim y Abele 1988). 
Informes previos en CR: playa Blanca, Guanacaste (Kim y Abele 1988).

Distribución: playa Blanca, Costa Rica, e isla Santa $\mathrm{Fe}$, islas Galápagos (Wicksten y Hendrickx 1992).

Comentarios: el holotipo procede de playa Blanca, Guanacaste (Kim y Abele 1988).

Alpheus antepaenultimus Kim \& Abele, 1988 Material: 39, Boca Guarumal, 23.XI.1995 (UCR 2108-04).

Hábitat: fondos lodosos y areno-lodosos (Kim y Abele 1988).

Informes previos en $\mathbb{C R}$ : Golfito, Costa Rica (Kim y Abele 1988).

Distribución: Golfito, Costa Rica, a Punta Paitilla, Panamá (Wicksten y Hendrickx 1992).

Comentarios: un paratipo procede de Golfito (Kim y Abele 1988).

Alpheus bellimanus Lockington, 1877

Material: 1ㅇ, Roca Sumergida, isla del Coco, 7.IV.1992 (UCR 1736-01). 10"29, Bajo Alcyone, isla del Coco, 2.IV.1992 (UCR 1741-01). 19, Roca Sucia, isla del Coco, 3.IV.1992 (UCR 1835-01).

Hábitat: de la zona más ba ja de entremareas hasta $300 \mathrm{~m}$, dragado con rocas y arena, lodo suave, grava fina o corales (Kim y Abele 1988).

Informes previos en $\mathbf{C R}$ : es primer informe para Costa Rica.

Distribución: de Monterrey, California, en la parte sur del golfo de California, islas Clarion y Socorro, el occidente de México, Panamá, Chile, Colombia y Galápagos (Kim y Abele 1988).

Alpheus bouvieri A. Milne Edwards, 1878

Material: ninguno.

Hábitat: coral muerto, sobre y entre rocas, arena y lodo, en arena, lodo y rocas, de la orilla a $1 \mathrm{~m}$ (Kim y Abele 1988)

Informes previos en CR: bahía Salinas, Guanacaste y Golfito, Puntarenas (Kim y Abele 1988).

Distribución: bahía Salinas, Guanacaste, Costa Rica, hasta Cabo San Francisco, Ecuador, en el Pacífico. En el lado Atlántico, de Bermuda al norte de Río Grande do Sul, Brasil; isla Ascensión; de isla Cabo Verde al Congo (Wicksten y Hendrickx 1992).

\section{Alpheus canalis Kim \& Abele, 1988}

Material: ninguno.

Hábitat: entre arena, lodo, roca y coral, de 0 a $37 \mathrm{~m}$ (Kim y Abele 1988).

Informes previos en $\mathbf{C R}$ : bahía Salinas, playa Blanca, playas del Coco, bahía Santa Elena, Guanacaste, y bahía Wafer, isla del Coco (Kim y Abele 1988).

Distribución: desde isla Tiburón, golfo de California, México, a isla Cabita, Colombia e islas Galápagos (Wicksten y Hendrickx 1992).

Alpheus cristulifrons Rathbun, 1900

Material: ninguno.

Hábitat: en bordes de arrecifes y asociado con rocas y coral incluyendo Pocillopora y Porites; de 0-1 m (Kim y Abele 1988).

Informes previos en CR: isla Tolinga, golfo de Nicoya (Kim y Abele 1988).

Distribución: puerto Escondido, golfo de California, México, a Taboga, Panamá (Wicksten y Hendrickx 1992).

Alpheus cylindrius Kingsley, 1878

Material: ninguno.

Hábitat: en arena, rocas y corales agrupados, de 0 a $37 \mathrm{~m}$ (Kim y Abele 1988).

Informes previos en CR: bahías Culebra y Santa Elena, Guanacaste (Kim y Abele 1988).

Distribución: del golfo de California, México, a isla Gorgona, Colombia e islas Galápagos. En el Atlántico de Bimini, Bahamas, a Bahía, Brasil (Wicksten y Hendrickx 1992).

Alpheus floridanus Kingsley, 1878

Material: 30'69, golfo de Nicoya, 5.XII.1993 (UCR 1996-02).

Hábitat: de la orilla hasta $37 \mathrm{~m}$, en lodo, coral, arena y rocas (Kim y Abele 1988)

Informes previos en $\mathbf{C R}$ : es primer informe para Costa Rica.

Distribución: en el Atlántico este, de Guinea hasta Congo e isla del Principe. En el Atlántico oeste del golfo de México al estado de Bahía, 
Brasil. En el Pacífico, del golfo de California, México, hasta Cabo San Francisco, Ecuador (Kim y Abele 1988).

Alpheus hamus Kim \& Abele, 1988

Material: ninguno.

Hábitat: en manglares (Kim y Abele 1988).

Informes previos en CR: Golfito, Puntarenas (Kim y Abele 1988).

Distribución: Golfito, Costa Rica; lado pacífico del Canal de Panamá, Panamá (Kim y Abele 1988).

Comentarios: el holotipo procede de Golfito, Costa Rica. Abele y Kim (1989) sinonimizaron de manera provisional esta especie con A. colombiensis Wicksten, 1988. En la lista presentada por Wicksten y Hendrickx (1991) aparecen ambas especies como válidas.

Alpheus hebes Kim \& Abele, 1988

Material: ninguno.

Hábitat: entre arena, lodo, arrecife rocoso y grupos de coral, de la orilla a $47 \mathrm{~m}$ (Kim y Abele 1988).

Informes previos en CR: bahías Chatham y Wafer, isla del Coco; bahías Salinas y Santa Elena, Guanacaste (Kim y Abele 1988).

Distribución: ensenada de San Francisco, Sonora, golfo de California, México, a islas Galápagos (Wicksten y Hendrickx 1992).

\section{Alpheus hyeyoungae Kim \& Abele, 1988}

Material: $40^{7} 6$ \%, isla del Caño, 24.III.1970 (UCR 2136-06).

Hábitat: rocas, arena de playa y coral (Kim y Abele 1988).

Informes previos en CR: playas del Coco, Guanacaste; isla Tolinga, golfo de Nicoya, Puntarenas (Kim y Abele 1988).

Distribución: de isla Tiburón, golfo de California, a islas Perlas, Panamá (Wicksten y Hendrickx 1992).

Alpheus leviusculus Dana, 1852

Material: ninguno.

Hábitat: en coral y rocas, de la orilla a $68 \mathrm{~m}$ (Wicksten 1983).
Informes previos en CR: Costa Rica (Wicksten 1983).

Distribución: del golfo de California, México, a islas Galápagos. En el Pacífico Indo-oeste, en islas Wabe y Cantón (Banner y Banner 1964).

Alpheus longinquus Kim \& Abele, 1988 Material: ninguno.

Hábitat: entre rocas, guijarros y colonias de coral (Kim y Abele 1988).

Informes previos en CR: bahía Wafer, isla del Coco, Puntarenas y bahía Santa Elena, Guanacaste (Kim y Abele 1988).

Distribución: bahía Agua Verde, golfo de California, México, a islas Galápagos (Wicksten y Hendrickx 1992).

Alpheus lottini Guérin-Méneville, 1829

Material: 3G, islas Palmitas, 27.III.1993 (UCR 2129-01). 40'7\%, isla del Caño, 6.IV.1970 (UCR 441-01). 19, isla del Caño, 24.I.1984 (UCR 1011-02). 10'1\%, isla del Caño, 23.II.1970 (UCR 405-01). 30"39, isla del Caño, 1.XI.1985 (UCR 541-01). 10' 1\%, isla del Caño, 26.I.1984 (UCR 2205-02).

Hábitat: comensal obligado de corales de la familia Pocilloporidae (Kim y Abele 1988).

Informes previos en $\mathrm{CR}$ : primer informe para Costa Rica.

Distribución: Nayarit, golfo de California, México, hasta Galápagos. En el Indo-Pacífico y Pacífico oeste, del Mar Rojo hasta Suráfrica. A lo largo del Océano Indico y el Pacífico tropical hasta Hawaii (Wicksten y Hendrickx 1992); Panamá (Glynn 1983).

\section{Alpheus malleator Dana, 1852}

Material: 10' 19, Cabo Blanco, 9.II.1993 (UCR 1871-01). 10", isla del Caño, 29.XI.1984 (UCR 1079-01).

Hábitat: arena fina y rocas, de 0 a $1 \mathrm{~m}$ de profundidad (Kim y Abele 1988).

Informes previos en CR: isla Tolinga, golfo de Nicoya, Puntarenas (Kim y Abele 1988).

Distribución: En el Atlántico este, de Senegal al Congo. En el Atlántico oeste, de Puerto Rico a São Paulo, Brasil. En el Pacífico en el 
golfo de California, en el Suroeste de México, Costa Rica, Ecuador, islas Malpelo y Galápagos (Kim y Abele 1988).

Alpheus martini Kim \& Abele, 1988

Material: 10 1\%, boca Guarumal, 23.X.1995 (UCR 2141-01).

Hábitat: de la orilla a $1 \mathrm{~m}$ de profundidad; entre arena, lodo arenoso, lodo, limo y rocas (Kim y Abele 1988).

Informes previos en $\mathbf{C R}$ : es primer informe para Costa Rica.

Distribución: del arrecife Whorehouse hasta punta Paitilla, Panamá (Wicksten y Hendrickx 1992).

Comentarios: se amplía la distribución geográfica hasta Sierpe, Puntarenas, Costa Rica.

Alpheus mazatlanicus Wicksten, 1983

Material: 20", frente a Chomes, 25.I.1969 (UCR 423-01). 19, punta Morales, 7.III. 1986 (UCR 1387-01). 10', Golfito, 5.II.1983 (UCR 443-01).

Hábitat: cerca de los manglares en la arena (Wicksten 1983)

Informes previos en $\mathbf{C R}$ : es primer informe para Costa Rica.

Distribución: estero de San Carlos, Sonora, México, hasta bahía Málaga, Colombia (Wicksten y Hendrickx 1992).

\section{Alpheus normanni Kingsley, 1878}

Material: ninguno.

Hábitat: sobre rocas, arena o conchas, debajo de la línea de marea hasta 73 m (Kim y Abele 1988).

Informes previos en $\mathbf{C R}$ : bahía Santa Elena, Guanacaste, Costa Rica (Kim y Abele 1988).

Distribución: bahía Cholla, Sonora, golfo de California, México, a islas Galápagos. En el Atlántico oeste, de Virginia, E.U.A. y Bermuda hasta la Península de Yucatán (Wicksten y Hendrickx 1992).

\section{Alpheus panamensis King, 1878}

Material: $20^{\circ} 2 \%$, punta Santa Elena, 14.X.1970 (UCR 968-08). 10 1\%, frente a isla Chora, Sámara, Guanacaste, 30.V.1997 (UCR
2207-12). 10", Cambutal, Dominical, 16.V.1995 (UCR 2123-02).

Hábitat: en rocas, lodo, arena y grava, de 0-1 $\mathrm{m}$ de profundidad (Kim y Abele 1988).

Informes previos en CR: playas del Coco, Guanacaste; isla Tolinga, golfo de Nicoya (Kim y Abele 1988).

Distribución: de playas del Coco, Costa Rica, hasta el "Mar Peruano" (Kim y Abele 1988).

\section{Alpheus pacificus Dana, 1852}

Material: ninguno.

Hábitat: en la zona entre mareas, viviendo bajo las rocas, en coral muerto en aguas hasta 20 $\mathrm{m}$; en la orilla entre guijarros y bancos de rocas (Kim y Abele 1988).

Informes previos en CR: bahía Wafer, isla del Coco, Puntarenas (Kim y Abele 1988).

Distribución: del golfo de California, México, a islas Galápagos. En el Pacífico Indo-oeste, del Mar Rojo hasta Madagascar y Hawai'i (Wicksten y Hendrickx 1992).

Alpheus paracrinitus Miers, 1881

Material: ninguno.

Hábitat: en rocas calcáreas, coral y colonias de coral. Sobre pastos marinos y entre colonias del coral Porites (Kim y Abele 1988).

Informes previos en $\mathbf{C R}$ : playa Blanca y playas del Coco, Guanacaste (Kim y Abele 1988). Distribución: Pantropical (Kim y Abele 1988).

Alpheus rostratus Kim \& Abele, 1988

Material: ninguno.

Hábitat: en roca y coral, de la orilla hasta 5.5 m (Kim y Abele 1988).

Informes previos en CR: bahía Santa Elena, Guanacaste (Kim y Abele 1988).

Distribución: isla Espíritu Santo, bahía de San Gabriel, golfo de California, México, hasta islas Galápagos (Wicksten y Hendrickx 1992).

Alpheus saxidomus Holthuis, 1980

Material: $\quad 120^{\circ}$, bahía Tamarindo, 28.VIII.1977 (UCR 2125-01). 10", punta Cóncava, Guanacaste, 14.VI.1992 (UCR 1482-01). 10 1\%, Sámara, Guanacaste, 30.V.1997 (UCR 
2207-03). 10 19, cabo Blanco, Puntarenas, 9.II.1993 (UCR 1880-07). 3ㅇ, cabo Blanco, 9.II.1993 (UCR 1871-11). 10'1\%, cabo Blanco, 10.III.1993 (UCR 1875-08). 19, cabo Blanco, 9.III.1 993 (UCR 1897-06). 107 1\%, punta Quepos, 17.II.1985 (UCR 2179-01). 10'2\%, isla del Caño, 25.I.1984 (UCR 2180-01). 10"39, isla del Caño, 25.I.1984 (UCR 1018-01). 10"1 1\%, isla del Caño, 28.IX.1984 (UCR 2126-01). 10', isla del Caño, 15.V.1985 (UCR 1950-01). 1\%, isla del Caño, 19.X.1984 (UCR 1074-01). 19, isla del Caño, X.1984 (UCR 1065-02). 19, isla del Caño, 17.X.1984 (UCR 1077-01). 10', isla del Coco, 16.VI.1994 (UCR 2119-01).

Hábitat: perfora rocas basálticas en la parte bajo de la zona de entremareas (Fischer 1981, 1990).

Informes previos en CR: playa Tamarindo, península de Nicoya (Holthuis 1980), a lo largo de la costa Pacífica (Fischer 1981).

Distribución: playa Tamarindo, península de Nicoya, Costa Rica, hasta bahías Octavia y Málaga e isla Gorgona, Colombia; islas Galápagos (Wicksten y Hendrickx 1992).

Comentarios: el holotipo procede de playa Tamarindo (Holthuis 1980). Se informa aquí de la presencia de esta especie en las islas del Caño y del Coco.

Alpheus sulcatus Kingsley, 1870

Material: $10^{\circ} 1 \%$, punta Santa Elena, Guanacaste, 14.X.1970 (UCR 968-08). 10", playa Tamarindo, Guanacaste, 27.I.1987 (UCR 213001). $20^{7} 19$, isla del Caño, 12.IV.1970 (UCR 543-01). 30'5\%, isla del Caño, 8.III.1970 (UCR 525-01). 140" 27\%, isla del Caño, 24.III.1970 (UCR 2136-01).

Hábitat: desde la orilla hasta $1.3 \mathrm{~m}$ en arena, rocas y coral (Kim y Abele 1988).

Informes previos en CR: playas del Coco, Guanacaste, e isla Tolinga, golfo de Nicoya (Kim y Abele 1988).

Distribución: en el Atlántico este, en São Tome y Congo. Indo-Pacífico, al este y sur de Africa, Mar Rojo, Australia y hasta las islas de la Sociedad. En el Pacífico este del golfo de California, México, hasta la isla Lobos de Tierra, Perú (Kim y Abele 1988).
Alpheus umbo Kim \& Abele, 1988

Material: ninguno.

Hábitat: en la orilla, entre rocas y coral (Kim y Abele 1988):

Informes previos en CR: bahía Santa Elena, Guanacaste (Kim y Abele 1988).

Distribución: Puerto Refugio, isla Angel de la Guarda, golfo de California, México, a Puerto Utria, Colombia (Wicksten y Hendrickx 1992).

Alpheus villus Kim \& Abele, 1988

Material: ninguno.

Hábitat: en la orilla en guijarros (Kim y Abele 1988). Informes previos en CR: bahía Wafer, isla del Coco, (Kim y Abele 1988).

Distribución: golfo de California, México; isla del Coco, Costa Rica e islas Secas, Panamá (Wicksten y Hendrickx 1992).

Alpheus websteri Kingsley, 1880

Material: $60^{\circ} 5$ 5, isla del Caño, 24.III.1970 (UCR 2136-02).

Hábitat: en rocas, playas de arena, pozos de marea entre rocas o coral. De la zona entre mareas hasta $6 \mathrm{~m}$ (Kim y Abele 1988).

Informes previos en CR: playas del Coco, Guanacaste e isla Tolinga, golfo de Nicoya (Abele y Kim 1989).

Distribución: en el Atlántico, de Bahamas, hasta la Península de Yucatán, México. En el Pacífico de Moro Colorado, Sonora, golfo de California, México, hasta islas Galápagos (Wicksten y Hendrickx 1992).

\section{Pomagnathus corallinus Chace, 1962}

Material: 2\%, Santa Elena, Guanacaste, 14.X.1970 (UCR 968-06).

Hábitat: zona de entre mareas y aguas someras, entre coral (Wicksten 1983).

Informes previos en CR: playa Blanca y bahía Santa Elena (Wicksten 1983).

Distribución: isla Cerralvo, golfo de California, México; islas Clarión, Clipperton, Malpelo y Galápagos (Wicksten y Hendrickx 1992).

Synalpheus biunguiculatus (Stimpson, 1860) Material: $10^{\circ} 5$ \% , isla del Caño, 25.II.1970 (UCR 541-03). 
Hábitat: entre rocas, guijarros o coral, de la zona entre mareas a $10 \mathrm{~m}$ (Wicksten 1983).

Informes previos en $\mathrm{CR}$ : es primer informe para Costa Rica.

Distribución: de Guaymas, Sonora, golfo de California, México, a Colombia; islas Clarión, Clipperton y Malpelo (Wicksten y Hendrickx 1992). En el Pacífico oeste, en Hawai'i (Wicksten 1983).

Synalpheus charon (Heller, 1861)

Material: 10 , isla del Caño, 25.II.1970 (UCR 541-01).

Hábitat: zona entre mareas, entre Pocillopora spp. (Wicksten 1983)

Informes previos en $\mathrm{CR}$ : es primer informe para Costa Rica.

Distribución: Mar Rojo, Océano Indico, Sur de China, al sur de Japón, Australia, Hawaii; del golfo de California, México, a isla Malpelo, Colombia; islas Clipperton y Clarión (Wicksten y Hendrickx 1992).

\section{Synalpheus digueti Coutière, 1909}

Material: ninguno.

Hábitat: zona entre mareas hasta $10 \mathrm{~m}$, entre rocas y coral (Wicksten 1983)

Informes previos en CR: bahía Santa Elena, Guanacaste, Costa Rica (Wicksten 1983)

Distribución: isla Tiburón, golfo de California, México, a islas Galápagos (Wicksten y Hendrickx 1992).

Synalpheus recessus Abele \& Kim, 1989

Material: $10^{\prime \prime} 39$, golfo de Nicoya, 16.XII.1993 (UCR 2010-08).

Hábitat: a $20 \mathrm{~m}$ de profundidad.

Informes previos en CR: golfo de Nicoya (Vargas et al. 1996).

Distribución: Panamá (Abele y Kim 1989) y golfo de Nicoya, Costa Rica (Vargas et al. 1996).

Synalpheus savjosei Coutière, 1909

Matenial: 10", Punta Morales, 21.II.1980 (UCR 950-01).

Hábitat: de la zcna entre marea a fondos duros someros sublitorales (Wicksten 1983).
Informes previos en CR: lado norte de punta Morales, golfo de Nicoya (Wicksten 1983).

Distribución: del golfo de California, México, hasta el golfo de Nicoya, Puntarenas (Wicksten 1983).

Familia HIPPOLYTIDAE Dana, 1852

Lysmata californica (Stimpson, 1866)

Informes previos en CR: el informe para los golfos de Nicoya y Dulce dado por Vargas et al. (1996) se debió a una mala identificación.

Thor algicola Wicksten, 1987

Material: 10", frente a isla Chora, Sámara, Guanacaste, 30.V.1997 (UCR 2207-13).

Habitat: entre Sargassum sp., guijaros, escombros, rocas, rocas y arena, lodo y arena, coral; de la orilla a $25 \mathrm{~m}$ (Wicksten 1987).

Informes previos: es primer informe para Costa Rica.

Distribución: de puerto Peñasco, Sonora, golfo de California, México, a bahía Piñas, Panamá (Wicksten y Hendrickx, 1992).

Thor amboinensis (De Man, 1888)

Material: niguno.

Habitat: siempre asociado con anemonas de mar de diferentes especies (Chace 1972); entre corales y rocas a $20 \mathrm{~m}$ (Wicksten 1990).

Informes previos: isla Manuelita, isla del Coco (Wicksten 1990).

Distribución: en el Atlántico tropical e IndoPacífico; isla del Coco y Panamá (Wicksten 1990).

Trachycaris restricta (A. Milne Edwards, 1878) Material: 2q, Santa Elena, Guanacaste, 14.IX.1970 (UCR 968-01).

Habitat: arena, rocas, lodo y fondos coralinos, de 0-100 m (Wicksten 1983).

Informes previos: es primer informe para Costa Rica.

Distribución: en el Atlántico este de islas Canarias a isla Santa Helena, Bermudas, Brasil. En el Pacífico este del golfo de California y Panamá (Wicksten 1990). 
Familia PROCESSIDAE Ortmann, 1896

Ambidexter panamensis Abele, 1972

Material: 39, Golfito, 1995 (UCR 2145-02)

Habitat: arena y lodo o rocas en aguas someras (Wicksten 1983). En raíces de Rhizophora mangle (esta publicación)

Informes previos: golfo de Nicoya (Wehrtmann 1990).

Distribución: San Diego, California; golfo de California; Panamá; islas Galápagos (Wicksten 1990). Costa Rica (Wehrtmann 1990).

Processa peruviana Wicksten, 1983

Material: 1\%, golfo de Nicoya, 14.XII.1993 (UCR 1980-07).

Hábitat: fondos arenosos, de 31-107 m de profundidad (Wicksten 1983).

Informes previos en CR: isla Manuelita, isla del Coco, Costa Rica (Wicksten 1983); golfo de Nicoya (Vargas et al. 1996).

Distribución: de Baja California, México, al norte de Mancora, Perú e islas Galápagos (Wicksten y Hendrickx 1992).

Comentarios: el holotipo procede de isla Manuelita, isla del Coco (Wicksten 1983).

Familia PANDALIDAE Haworth, 1825

Heterocarpus affinis Faxon, 1893

Material: $10^{\prime} 1 \%$, costa pacífica, s.f. (UCR 1807-05).

Hábitat: en el talud continental de 1,208 a 1,244 m (Méndez 1981).

Informes previos en CR: Costa Rica (Méndez 1981).

Distribución: mar afuera de islas Tres Marias, Nayarit, México, hasta mar afuera de Mancora, Perú (Hendrickx y Wicksten 1989).

\section{Heterocarpus vicarius Faxon, 1893}

Material: 3q, cabo Blanco, Puntarenas, 11.VIII.1981 (UCR 1434-01). 180'199, golfo de Nicoya, 15.XII.1993 (UCR 1982-02). 30" 39, golfo Dulce, 11.XII.1993 (UCR 2005-02). Hábitat: casi estrictamente bentónico, entre 62 y $1,454 \mathrm{~m}$ de profundidad (Hendrickx y Wicksten 1989)
Informes previos en CR: Costa Rica (Méndez 1981). Golfos de Nicoya y Dulce, bahía Coronado (Vargas et al. 1996).

Distribución: desde Baja California, México, hasta Mollendo, Perú (Hendrickx y Wicksten 1989).

Pantomus affinis Chase, 1937

Material: 4\%, golfo de Nicoya, 14 18.XII.1993 (UCR 19881-02). 20'14\%, golfo Dulce, 11.XII.1993 (UCR 2009-01). 50' 20\%, golfo Dulce, 11.XII.1993 (UCR 2005-01).

Hábitat: exclusivamente bentónico, en fondos lodosos, fangosos con arena fina, arenosos con piedras y esquisto, arenosos y limosos en condiciones de oxigenación y temperatura relativamente entre 3 y 744 m (Hendrickx \& Wicksten 1989).

Informes previos en CR: golfo Dulce, Costa Rica (Hendrickx y Wicksten 1989), golfos de Nicoya y Dulce (Vargas et al. 1996).

Distribución: del golfo de California, México, a la isla Lobos de Tierra, Perú (Wicksten y Hendrickx 1992).

\section{Plesionika mexicana Chase, 1937}

Material: $60^{\top} 3$, golfo de Nicoya, 14.II.1994 (UCR 2011-01). 20'99, golfo de Nicoya, 13.II.1994 (UCR 1984-02).

Hábitat: en fondos lodosos, arenosos, conchíferos y duros, entre 28 y $258 \mathrm{~m}$ (Wicksten 1983).

Informes previos en CR: golfos de Nicoya y Dulce, bahía de Coronado (Vargas et al. 1996). Distribución: de Redondo Beach, California, E.U.A., hasta banco Mancora, Perú e islas Galápagos (Wicksten y Hendrickx 1992).

\section{Familia CRANGONIDAE Haworth, 1825}

\section{Sclerocrangon atrox Faxon, 1893}

Material: 1 Q , costa pacífica, s.f. (UCR 1807-08). Hábitat: sobre el talud continental, entre 800 y $1250 \mathrm{~m}$ (Hendrickx 1995b).

Informes previos en CR: es primer informe para Costa Rica.

Distribución: de islas Tres María, México a Perú (Wicksten 1989) 


\section{Familia GLYPHOCRANGONIDAE}

Smith, 1884

Glyphocrangon alata Faxon, 1893

Material: $10^{7} 6 \%$, costa pacífica, s.f. (UCR 1807-04).

Hábitat: bentónico sobre el talud continental (Méndez 1981). De 600 a 1,335 m (Chace 1984).

Informes previos en $\mathbf{C R}$ : es primer informe para Costa Rica.

Distribución: mar afuera de Acapulco, Guerrero, México, hasta mar afuera de Valparaíso, Chile (Wicksten y Hendrickx 1992).

Glyphocrangon sicaria Faxon, 1893

Material: ninguno

Hábitat: de 1,454 a 3,310 m (Wicksten 1989). Informes previos en $\mathbf{C R}$ : al sur de punta Guiones, Guanacaste (Wicksten 1979).

Distribución: del sur de punta Guiones, Guanacaste, Costa Rica, al golfo de Panamá (Wicksten y Hendrickx 1992).

Glyphocrangon vicaria Faxon, 1893

Material: ninguno.

Hábitat: bentónico entre 1375 y 3880 m (Hendrickx 1995b).

Informes previos en CR: mar afuera de punta Guiones, Costa Rica (Wicksten 1979).

Distribución: base de San Clemente, sureste de California, E.U.A. a islas Galápagos (Wicksten y Hendrickx 1992).

\section{ASTACIDEA Latreille, 1803}

\section{Familia NEPHROPIDAE Dana, 1852}

Nephropsis occidentalis Faxon, 1893

Material: $20^{\circ} 4 \%$, costa pacífica, s.f. (UCR 1807-01).

Hábitat: sobre fondos blandos en aguas profundas, entre 270 y 1200 m (Hendrickx 1995b).

Informes previos en CR: es primer informe para Costa Rica.

Distribución: costa oeste de Baja California y mar afuera de bahía Santa María, Sinaloa, golfo de California, México a Valparaíso, Chile (Hendrickx 1995b).
THALASSINIDEA Latreille, 1831

Familia AXIIDAE Huxley, 1879

Axiopsis serratifrons (A. Milne Edwards, 1873) Material: 1\%, isla San José, islas Murciélago, Guanacaste, 7.XII.1969 (UCR 544-02). 1\%, punta Esmeralda, Bahía Culebra, 28.VIII.1997 (UCR 2006-01)

Hábitat: más allá del nivel más bajo de la marea pero cercano a ésta, entre piedras y rocas (Lemaitre y Ramos 1992).

Informes previos en $\mathbf{C R}$ : es primer informe para Costa Rica.

Distribución: isla Gorgona en el Pacífico este; el Indo-Pacífico incluyendo el Mar Rojo; en el Atlántico oeste de Florida a Belize, incluyendo Bermuda y en el Atlántico Sur en isla Ascención (Lemaitre y Ramos 1992).

Comentario: se amplía la distribución geográfica en el Pacífico este hacia el norte a bahía Culebra, Guanacaste, Costa Rica.

Familia CALLIANASSIDAE Dana, 1852

Biffarius fragilis (Biffar, 1970)

Material: 10", golfo de Nicoya, 10.VII.1980 (UCR 2003-01).

Hábitat: cerca de la costa (Manning y Felder 1991)

Informes previos en $\mathbf{C R}$ : es primer informe para Costa Rica.

Distribución: Atlántico noroeste (Manning y Felder 1991). En el Pacífico este, en California e islas Galápagos (Biffar 1972).

Callichirus seilacheri Bott, 1955

Material: $20^{\circ} 6 \%$, puerto Caldera, 28.IV.1998 (UCR 2246-01). 320 199, puerto Caldera, 28.IV.1998 (UCR 2246-02). 10", manglar de Tivives, Puntarenas, IX.1995 (UCR 2156-01).

Hábitat: zona entre mareas (Lemaitre y Ramos 1992).

Informes previos en $\mathbf{C R}$ : es primer informe para Costa Rica.

Distribución: del Salvador a Chile (Lemaitre y Ramos 1992). 
Lepidophthalmus bocourti (A. Milne Edwards, 1870)

Material: $10^{7} 2 \%$, estero en playa Ostional, Guanacaste, 13.X.1982 (UCR 1612-01). 1\%, Ostional, 4.X.1982 (UCR 1491-01). 507 69, Colorado de Abangares, 14.XII.1981 (UCR 161301). $30^{7} 10$ \%, Chomes, Puntarenas, VI.1991 (UCR 1509-01). 29, manglar de boca Guarumal, Sierpe, 23.XI.1995 (UCR 2108-03).

Hábitat: zona entre mareas, cerca de corrientes de agua dulce en madrigueras en lodo o sedimentos finos de origen basáltico (Lemaitre y Ramos 1992).

Informes previos en CR: es primer informe para Costa Rica.

Distribución: costa Pacífica de México a Colombia (Lemaitre y Ramos 1992).

\section{Familia CALLIANIDEIDAE Kossmann, 1880}

\section{Callianidea laevicauda Gill, 1859}

Material: $10^{\prime}$, playa Blanca, Parque Nacional Santa Rosa, 16.II.1995 (2020-08).

Hábitat: de la zona entre mareas a aguas sublitorales someras (Lemaitre y Ramos 1992).

Informes previos en $\mathrm{CR}$ : es primer informe para Costa Rica.

Distribución: en el Pacífico de Baja California e isla Socorro, México, a Colombia e islas Galápagos. En el Mar Caribe (Lemaitre y Ramos 1992).

\section{Familia UPOGEBIIDAE Borradaile, 1903}

Pomatogebia cocosia (Williams, 1986)

Material: 10", isla del Coco, 1988 (UCR 173501). 19, isla del Coco, III.1989 (UCR 1932-03).

Hábitat: abajo de la línea de marea (Lemaitre y Ramos 1992).

Informes previos en CR: isla del Coco (Williams 1986).

Distribución: isla del Coco, Costa Rica (Williams 1986).

Comentarios: el holotipo procede de la isla del Coco (Williams 1986). Especie endémica a la isla del Coco.
Pomatogebia rugosa (Lockington, 1878)

Material: $1 \%$, punta Uvita, Puntarenas, 24.II.1994 (UCR 1081-01). 19, isla del Caño, 1988 (UCR 1925-01). 10̛ 2\%, Sándalo, golfo Dulce, 16.III.1996 (UCR 2200-01). $10^{7} 2 \%$, golfo Dulce, 12.III.1989 (UCR 1770-01).

Hábitat: abajo de la línea de marea hasta alrededor de $18 \mathrm{~m}$, perforando el coral Porites lobata (Cortés 1991).

Informes previos en CR: golfo Dulce (Cortés 1991, Fonseca y Cortés 1998).

Distribución: de Baja California, México, hasta Colombia (Lemaitre y Ramos 1992).

Upogebia burkenrroadi Williams, 1986

Material: $10^{7} 2 \%$, punta Pitahaya, Guanacaste, 15.VI.1991 (UCR 1708-01.

Hábitat: viven en huecos recubiertos con lodo en su interior de $5 \mathrm{~mm} \times 5 \mathrm{~mm}$., la conexión al exterior está cubierta con arena y algunas algas (Williams 1997).

Informes previos en $\mathrm{CR}$ : punta Pitahaya, Guanacaste (Williams 1997).

Distribución: Sonora, México; punta Pitahaya, Guanacaste, Costa Rica (Williams 1997).

Upogebia dawsoni Williams, 1986

Material: ninguno.

Hábitat: la mayoría de los especímenes conocidos ha sido recolectada en estuarios (Williams 1986).

Informes previos en CR: bahía Potrero Grande, Guanacaste e isla San Lucas, golfo de Nicoya (Williams 1986).

Distribución: de Baja California, México, a la zona del Canal, Panamá (Williams 1986).

Upogebia jonesi Williams, 1986

Material: ninguno.

Hábitat: en arena y lodo, hasta $73 \mathrm{~m}$ (Williams 1986).

Informes previos en CR: golfo de Nicoya (Williams 1986).

Distribución: costa pacífica de México a Panamá (William 1986). 
Upogebia maccraryae Williams, 1986

Material: $10^{\prime} 1$, estero punta Morales, 1995 (UCR 2142-06).

Hábitat: en manglares, fondos lodosos cubiertos con hojas y en madrigueras en madera podrida (Williams 1986).

Informes previos en CR: primer informe para Costa Rica.

Distribución: del Salvador a Ecuador (Williams 1986).

\section{Upogebia spinigera (Smith, 1871)}

Material: 20’ 2\%, cabo Blanco, 27.X.1992 (UCR 1976-08). 10’3 3, punta Morales, 3.V.1991 (UCR 1710-01). 1, punta Morales, 21-II-1980 (UCR 952-01). 1, punta Morales, 11.VIII.1984 (UCR 1060-03). 2q 1juv., punta Morales, 1995 (UCR 2142-05). 1 \% 1juv., punta Morales, 2.VIII.1996 (UCR 2196-01). 10' 2\%, punta Morales, 24.V.1997 (UCR 2195-01). 110'169, estero Guarumal, 23.XI.1995 (UCR 2108-01). 1\%, península de Osa, 18.XII.1990 (UCR1727-01). 2ㅇ, puerto Jiménez, 31.I.1997).

Hábitat: se ha recolectado en una gran variedad de hábitats arena, rocas, lodo y en pozos de marea principalmente (Williams 1986).

Informes previos en CR: lado norte de Punta Morales e isla San Lucas, golfo de Nicoya (Williams 1986).

Distribución: del golfo de Fonseca, El Salvador, a Ecuador (Williams 1986).

\section{Upogebia vargasae Williams, 1997}

Material: $30^{7} 13$ q 1juv., manglar de TérrabaSierpe, Puntarenas, 23.XI.1995 (UCR 210802). $20^{\prime} 3$ (paratipos), manglar de TérrabaSierpe, Puntarenas, 23.XI.1995 (UCR 210802-01).

Hábitat: cuevas en bancos verticales de lodo expuestos durante marea baja; asociada con $U$. spinigera

Informes previos en CR: manglar de TérrabaSierpe, Puntarenas (Williams 1997).

Distribución: conocido solo de la localidad tipo, Boca Guarumal, Manglar de TérrabaSierpe, Puntarenas, Costa Rica (Williams 1997).
Comentarios: el lote de organismos citado es el mismo que se uso para describir la especie.

PALINURA Latreille, 1803

Familia POLYCHELIDAE Wood-Mason, 1874

Stereomastis sculpta pacifica(Faxon 189.3)

Material: 5 ㅇ y 2 larvas, costa pacífica, s.f. (UCR 1907-03).

Hábitat: adultos en fondos suaves entre 10003692 m; larvas nectónicas (Wicksten, 1989).

Informes previos: Costa Rica (Wicksten 1981).

Distribucion: de isla San Clemente, California, E.U.A. a Valparaíso, Chile, incluyendo mar afuera de islas Tres Marías, Nayarit, golfo de California, México (Hendrickx 1995b).

\section{Familia PALINURIDAE Latreille, 1803}

\section{Panulirus gracilis Streets, 1871}

Material: $10^{\prime}$ 4juv., Tamarindo, Guanacaste, 27.I.1967 (UCR 1505-01). 1오, golfo de Nicoya, 19.VI.1972 (UCR 633-01). 10', Caldera, 11.II.1964 (UCR 1328-01). $10^{\circ} 3$ \%, frente a río Tárcoles, 8.IX.1970 (UCR 597-01). 207, 2 km afuera de la desembocadura del río Tárcoles, 8-11.IX.1970 (UCR 9964-01).

Hábitat: en zonas rocosas, entre rocas y grietas, generalmente de 15 a $22 \mathrm{~m}$ en aguas relativamente turbias (Hendrickx 1995b).

Informes previos en CR: isla del Coco (Hertlein 1963).

Distribución: desde bahía Magdalena en la costa oeste de Baja California Sur, La Paz Baja California Sur y Mazatlán, Sinaloa, golfo de California, México, hasta Paita, Perú (Hendrickx 1995a).

\section{Panulirus penicillatus Olivier, 1791}

Material: ninguno.

Hábitat: vive en zonas rocosas y arrecifes coralinos generalmente en profundidades no mayores de $8 \mathrm{~m}$. Prefiere aguas agitadas y claras (Hendrickx 1995b).

Informes previos en CR: isla del Coco (Hertlein 1963). 
Distribución: desde Cerritos hasta Guerrero, México; e islas Socorro, Clipperton, Coco, Malpelo y Galápagos (Hendrickx 1995a). Indo-Pacífico (Lemaitre y Alvares León 1992).

Familia SCYLLARIDAE Latreille, 1825

\section{Evibacus princeps S.I. Smith, 1869}

Material: 10̛, golfo de Nicoya, 9.I.1969 (UCR 1302-01). 10", golfo de Nicoya, 11.IV.1980 (UCR 1696-01). 10', golfo de Nicoya, 79.III.1967 (UCR 117-01). 19, bahía Caldera, 3.X.1965 (UCR 116-01). 1juv., bahía Ballena, 14.XII.1993 (UCR 1980-10).

Hábitat: asociada principalmente a fondos arenosos pero también en lodosos, limosos y ocasionalmente rocosos y mixtos, entre 3 y 90 m (Hendrickx . 1995b).

Informes previos en $\mathbf{C R}$ : golfo de Nicoya (Maurer et al. 1984, Vargas et al. 1996).

Distribución: desde Puerto Peñasco, Sonora y Puerto Escondido, Baja California Sur, golfo de California, México, hasta Mancora, Perú (Hendrickx 1995a).

\section{DISCUSIÓN}

La lista presentada en este trabajo incluye un total de 117 especies de camarones y langostas. De esos, 27 especies (23\%) representan nuevos informes para la costa pacífica de Costa Rica. Cinco especies representan ampliaciones de ámbito; tres de éstas son significativas: Pontonia simplex (Holthuis, 1951), amplía su distribución desde bahía Tenacatitán, México hasta bahía Culebra, Guanacaste; Veleronia serratifrons (Holthuis, 1951) amplía su distribución al norte desde La Libertad, Ecuador hasta Sámara, Guanacaste; y Axiopsis serratifrons (A. Milne Edwards, 1873) también se extiende al norte desde la isla Gorgona, Colombia hasta bahía Culebra, Guanacaste.

Nueve especies fueron descritas con material recolectado en Costa Rica: Sicyonia affinis, de isla del Coco (Faxon 1893); Macrobrachium hancoki, del río Barranca, Esparta, Puntarenas (Holthuis 1952); Periclimenes spinosus, recolectada al sur de islas Viradores, bahía Culebra,
Guanacaste (Holthuis 1951); Pontonia spighti, de playas del Coco, Guanacaste (Fujino 1972); Alpheus aequus, de playa Blanca, Guanacaste (Kim y Abele 1988); Alpheus hamus, Golfito, Costa Rica (Kim y Abele 1988); Alpheus saxidomus, de playa Tamarindo (Holthuis 1980); Processa peruviana, recolectado en la isla Manuelita, isla del Coco (Wicksten 1983); y Pomatogebia cocosia, también de la isla del Coco (Williams 1986). Dos especies son todavía consideradas endémicas de Costa Rica: Pontonia spighti, recolectado en playas del Coco (Fujimo 1972) y Pomatogebia cocosia, de la isla del Coco (Williams 1986).

Wicksten y Hendrickx (1992), presentaron una lista en la que se informa de 227 especies para el Pacífico Este Tropical (PET) 39 de Penaeoidea y 188 de Caridea. Para Costa Rica, se informan 28 especies de Penaeoidea y 70 de Caridea. Comparando la información de Wicksten y Hendrickx (1992) para el PET con lo informado para Costa Rica, contamos con 43\% de las especies de Penaeoidea y Caridea. Debido a la diversidad de ambientes en la costa Pacífica de Costa Rica, a los pocos muestreos en aguas profundas y la distribución de algunas especies, es probable que ese porcentaje sea una subestimación de la diversidad de estos grupos de crustáceos en Costa Rica.

Cabe señalar también que solo un $72 \%$ de las especies informadas aquí están representadas en la colección de crustáceos del Museo de Zoología, una razón más para motivar la recolección de material y fomentar la idea de repatriar colecciones de Costa Rica presentes en museos zoológicos en el exterior.

\section{AGRADECIMIENTOS}

Le agradecemos a Allison Villalobos la ayuda recibida para realizar esta recopilación; a Kay Hale, directora de la biblioteca de RSMAS, Universidad de Miami, el apoyo con literatura. A Rafael Lemaitre y Ana Dittel, por el apoyo con literatura y la revisión del manuscrito; a Michel Hendrickx por las sugerencias al manuscrito, y a la Escuela de Biología por el apoyo al Museo de Zoología. Esta publicación se pudo 
realizar gracias al aporte económico de la Vicerrectoría de Investigación (proyectos 808-92237, 808-96-601), del CONICIT (proyecto 90326-BID), del CIMAR, y de la Escuela de Biología. Esta es una contribución del Museo de Zoología, Escuela de Biología, y del Centro de Investigación en Ciencias del Mar y Limnología (CIMAR), Universidad de Costa Rica.

\section{RESUMEN}

En el Pacífico de Costa Rica se ha encontrado 117 especies de camarones y langostas. Estas se ubican en el Suborden Dendrobranchiata (Penaeoidea, 25 spp.; Sergestoidea, 1 sp.); y en el Suborden Pleocyemata (Caridea, 73 spp.; Astacidea, $1 \mathrm{sp}$; Thalassinidea, $13 \mathrm{spp}$. y Palinura, 4 spp.). Veintisiete especies (23\%) son nuevos infomes para Costa Rica. Cinco informes representan ampliaciones de ámbito, tres de importancia: Pontonia simplex (Holthuis, 1951) se extiende la distribución desde la bahía Tenacatitán, México hasta bahía Culebra, Guanacaste; Veleronia serratifrons (Holthuis, 1951) se amplia la distribución al norte desde La Libertad, Ecuador hasta Sámara, Guanacaste y Axiopsis serratifrons (A. Milne Edwards, 1873) también al norte desde la isla Gorgona, Colombia hasta bahía Culebra, Guanacaste. Nueve especies fueron descritas con material recolectado en Costa Rica y dos son endémicas. $43 \%$ de las especies de camarones del Pacífico este se encuentran en la costa Pacífica de Costa Rica. El total de especies indicado aquí es probablemente una subestimación de la diversidad de estos grupos en el Pacífico de Costa Rica.

\section{REFERENCIAS}

Abele, L.G. \& W. Kim. 1986. An illustrated guide to the marine decapod crustaceans of Florida. Dept. Environ. Reg. Tech. Ser. 8: 1-326.

Abele, L.G. \& W. Kim. 1989. The decapod crustaceans of the Panama Canal. Smithson. Contr. Zool. 482: 1-50.

Banner, A.H. \& D.M. Banner. 1964. Contributions to the knowledge of the alpheid shrimps of the Pacific Ocean, IX. Collections from the Phoenix and Line islands. Pac. Sci. 18: 83-100.

Biffar, T.A. 1972. A study of the eastern Pacific representatives of the genus Callianassa (Crustacea, Decapoda, Callianassidae). Disertación Doctoral, University of Miami, Miami, Florida. 281p.

Boone, L. 1931. Anomuran, Macruran Crustacea from Panama and Canal Zone. Bull. Am. Mus. Nat. Hist. 63: $1-180$.
Bowman, T.E. \& L.G. Abele. 1982. Classification of the recent crustacea: 1-27. In: Bliss, D.E. \& L.G. Abele (eds). The biology of crustacea. Vol.1 Systematics, the fossil record and biogeography. Academic, Nueva York.

Burkenroad, M.D. 1938. The Templeton Crocker Expedition. XIII. Penaeidae from the region of Lower California and Clarion Island, with description of four new species. Zoologica 23: 59-91.

Cabrera P., J. y Y. Solano L. 1996. Tamaños y frecuencias de Pontonia margarita (Crustacea: Palaemonidae) asociadas a Pinctada mazatlanica (Bivalvia: Pteridae), Costa Rica. Rev. Biol. Trop. 44: 915-917.

Cedeño, F. 1986. Contribución al conocimiento de los camarones de agua dulce de Costa Rica. Tesis de Licenciatura, Escuela de Biología, Universidad de Costa Rica. 104 p.

Chace, F.A., Jr. 1972. The shrimps of the SmithsonianBredin Caribbean Expeditions with a summary of the West Indian shallow-water species (Crustacea: Decapoda: Natantia). Smithson. Contr. Zool. 98: 1-179.

Chace, F.A., Jr. 1984. The caridean shrimps (Crustacea: Decapoda) of the Albatros Philippine Expedition, 1907-1910, Part. 2: Families Glyphocrangonidae and Crangonidae. Smithson. Contr. Zool. 397: 1-63.

Cortés, J. 1991. Los arrecifes coralinos del Golfo Dulce, Costa Rica: Aspectos geológicos. Rev. Geol. Amér. Central 3: 15-24

Cortés, J. 1996. Biodiversidad marina de Costa Rica: Filo Porifera. Rev. Biol. Trop. 44: 911-914.

Cortés, J. 1996-1997. Biodiversidad marina de Costa Rica: Filo Cnidaria. Rev. Biol. Trop. 44(3)/45(1): 323-334.

Faxon, W. 1893. Preliminary descriptions of new species of Crustacea. Bull. Mus. Comp. Zool. 24: 149-220.

Faxon, W. 1895. Reports on an exploration off the west coast of México, Central and South America, and off the Galápagos Islands by the U.S. Fish Commission Steamer "Albatros", during 1891.XV. The stalk-eyed Crustacea. Mem. Mus. Com. Zool. 18: 1292.

Fischer, R. 1981. Bioerosion of basalt of the Pacific coast of Costa Rica. Senckenberg. Marit. 13: 1-41.

Fischer, R. 1990. Biogenetic and non biogenetic determined morphologies of the Costa Rican Pacific coast. Z. Geomoph. N.F. 34: 313-321. 
Fonseca E., A.C. \& J. Cortés. 1998. Coral borers of the eastern Pacific: the sipunculan Aspidosiphon (A.) elegans and the crustacean Pomatogebia rugosa. Pac. Sci. 52: 170-175.

Fujino, T. 1972. A new pontoniinid shrimp, Pontonia spighti sp new., associated with a newly described ascidian from the pacific coast of Costa Rica (Decapoda, Natantia, Pontoniinae). Publ. Seto Mar. Biol. Lab. 19: 293-301.

Glynn, P.W. 1983. Crustacean symbionts and the defense of corals: coevolution on the reef?: p. 111-178. In: M.H. Nitecki (ed.), Coevolution. University of Chicago, Chicago.

Hendrickx, M.E. 1984. The species of Sicyonia H. Milne Edwards (Crustacea: Penaeoidea) of the Gulf of California, Mexico, with a key for their identification and a note on their zoogeography. Rev. Biol. Trop. 32: $279-298$.

Hendrickx, M.E. 1995a. Checklist of lobster-like decapod crustaceans (Crustacea: Decapoda: Thalassinidea, Astacidea and Palinura) from the eastern tropical Pacific. Anales Inst. Biol. Univ. Nac. Autón. México, Ser. Zool. 66: 151-163.

Hendrickx, M.E. 1995b. Camarones: 417-537. In: Fischer, W.; F. Krupp, W. Schneider, C. Sommer, K.E. Carpenter \& U.H. Niem (eds.), Guía FAO para la identificación de especies para los fines de la pesca. Pacífico Centro-oriental. Vol. 1. Plantas e Invertebrados. Organizacion de las Naciones Unidas para la alimentación y la Agricultura, Roma.

Hendrickx, M.E. 1996. Los camarones Penaeoidea (Crustacea: Decapoda: Dendrobranchiata) del Pacífico mexicano. Comisión Nacional para el Conocimiento y uso de la Biodiversidad (Conabio) e Instituto de Cienicas del Mar y Limnología, Universidad Nacional Autónoma de México. 147p.

Hendrickx, M.E. \& F.D. Estrada Navarrete. 1996. Los camarones pelágicos del Pacífico Mexicano (Crustacea: Dendrobranchiata y Caridea). Comisión Nacional para el conocimiento y uso de la biodiversidad e Instituto de Ciencias del mar y limnología, Universidad Autónoma de México. 157p.

Hendrickx, M.E. \& M.K. Wicksten. 1989. Los Pandalidae (Crustacea: Caridea) del Pacífico mexicano, con una clave para su identificación. Caldasia (Colombia) 16: 71-86.

Hertlein, L.G. 1963. Contribution to the biogeography of Cocos Island, icluding a bibliography. Proc. Calif. Acad. Sci. 32: 219-289.
Holthuis, L.B. 1951. A general revision of the Palaemonidae (Crustacea: Decapoda: Natantia) of the Americas. I. The subfamilies Euryrhynchinae and Pontoniinae. Occ. Papers Allan Hancock Foundation 11: 1-332.

Holthuis, L.B. 1952. A general revision of the Palaemonidae (Crustacea: Decapoda: Natantia) of the Americas, Part. II. The subf amily Palaemoninae. Occ. Papers Allan Hancock Foundation 12: 1-396.

Holthuis, L.B. 1980. Alpheus saxidomus new species, a rock boring snapping shrimp from the Pacific coast of, Costa Rica, with notes on Alpheus simus Guérin-Méneville, 1856. Zool. Medeling. 55: 47-58.

Kim W. \& L.G. Abele. 1988. The snapping shrimp genus Alpheus from the Eastern Pacific (Decapoda: Caridea: Alpheidae). Smithson. Contr. Zool. 454: 1119.

Kitani, H. 1997. Postlarval morphology of the western white shrimp Penaeus occidentales (Crustacea: Penaeidae). Rev. Biol. Trop. 44: 627-630.

Lemaitre, R. \& R. Alvarez-León. 1992. Crustáceos decápodos del Pacífico colombiano: Lista de especies y consideraciones zoogeográficas. An. Inst. Invest. Mar. Punta Betín 21: 33-76.

Lemaitre, R. \& G.E. Ramos. 1992. A collection of Thalassinidea (Crustacea: Decapoda) from the Pacific coast of Colombia, with description of a new species and a checklist of eastern Pacific species. Proc. Biol. Soc. Wash. 105: 343-358.

Manning, R.B. \& D.L. Felder. 1991. Revision of the american Callianassidae (Crustacea: Decapoda: Thalassinidea). Proc. Biol. Soc. Wash. 104: 7764-792.

Maurer, D., C.E. Epifanio, H.K. Dean, S. Howe, J.A Vargas, A.I. Dittel y M.M. Murillo. 1984. Benthic invertebrates of a tropical estuary: Gulf of Nicoya, Costa Rica. J. Nat. Hist. 18: 47-61.

Méndez, M. 1981. Claves de identificación y distribución de los langostinos y camarones (Crustacea: Decapoda) del mar y los ríos de la costa del Perú. Bol. Inst. Mar Perú-Callao 5: 1-170.

Pérez-Farfante, I. 1975. Spermatophores and thelyca of the American white shrimps, genus Penaeus, subgenus Litopenaeus. Fish. Bull. 73: 463-486.

Pérez-Farfante, I. 1977. American solenocerid shrimps of the genera Hymenopenaeus, Haliporides, Pleoticus, Hadropenaeus new genus, and Mesopenaeus new genus. Fish. Bull. 75: 261-346. 
Pérez-Farfante, I. 1985. The rock shrimp genus Sicyonia (Crustacea: Decapoda: Penaeoidea) in the eastern Pacific. Fish. Bull. 83: 1-79.

Pérez-Farfante, I. 1988. Ilustrated key to penaeoid shrimp of commerce in the Americas. NOAA Tech. Rep. NMFS 64: 1-32.

Pérez-Farfante, I. \& B. Kensley. 1997. Penaeoid and Sergestoid shrimps and prawns of the world. Mém. Mus. Nat. Hist. Nat. (Paris) 175: 1-233.

Pesta, O. 1931. Ergebnisse der Osterreichsvchen biologischen Costa Rica Expedition 1930. I. Teil. Crustacea Decapoda aus Costa Rica. Ann. Naturhist. Mus. Wien 45: 173-181.

Ríos, R. 1986. Caridean shrimps of the gulf of Calif ornia V. New records of species belonging to the subf amily Pontomiinae (Crustacea: Decapoda: Palaemonidae). Proc. Biol. Soc. Wash. 99: 429-434.

Vargas, R. \& J. Cortés. 1997. Biodiversidad marina de Costa Rica: Orden Stomatopoda (Crustacea: Malacostraca: Hoplocarida). Rev. Biol. Trop. 45: 1531-1539.

Vargas, R. \& J. Cortés. 1999. Biodiversidad marina de Costa Rica: Crustacea: Decapoda (Penaeoidea, Sergestoidea, Stenopodidae, Caridea, Thalassinidea y Palinura) del Caribe. Rev. Biol. Trop. 47:877-885.

Vargas, R., S. Jessi \& M. Castro. 1996. Checklist of crustaceans (Decapoda and Stomatopoda), collected during the Victor Hensen Costa Rica expedition (1993/1994). Rev. Biol. Trop. 44, Suppl.3: 97-102.

Wehrtmann, I.S. 1990. Distribution and reproduction of Ambidexter panamense and Palaemonetes schmitti in Pacific Costa Rica (Crustacea, Decapoda). Rev. Biol. Trop. 38: 327-329.
Wicksten, M.K. 1979. New records of the species of Glyphocrangon in the northeastern Pacific Ocean (Caridea: Glyphocrangonidae). Proc. Biol. Soc. Wash. 92: 217-224.

Wicksten, M.K. 1981. New records of Steromastis sculpta pacifica (Faxon) (Decapoda: Polychelidae) in the eastern Pacific Ocean. Proc. Biol. Soc. Wash. 93: 914-919.

Wicksten, M.K. 1983. Shallow water caridean shrimps of the Gulf of California, México. Allan Hancock Monog. Mar. Biol. 13: 1-59.

Wicksten, M.K. 1987. A new species of Hippolytid shrimp from the west coast of Mexico. Bull. Southern California Acad. Sci. 86: 27-33.

Wicksten, M.K. 1989. Ranges of off shore decapod crustaceans in the eastern Pacific Ocean. Trans. San Diego Soc. Nat. Hist. 21: 291-316.

Wicksten, M.K. 1990. Key to the hippolytid shrimp of the Eastern Pacific Ocean. Fish. Bull. 88: 587598.

Wicksten, M.K.\& M.E. Hendrickx. 1992. Checklist of Penaeoid and Caridean Shrimps (Decapoda: Penaeoidea, Caridea) from the eastern tropical Pacific. Proc. San Diego Soc. Nat. Hist. 9: 1-11.

Williams, A. 1986. Mud shrimps, Upogebia, from the eastern Pacific (Thalassinoidea: Upogebiidae). San Diego Soc. Nat. Hist. Memoir 14: 1-60.

Williams, A. 1997. Two new species and a range extension of mud shrimps, Upogebia, from Pacific Costa Rica and Mexico (Decapoda: Thalassinidea: Upogebiidae). Proc. Biol. Soc. Wash. 110: 617623. 